\title{
Antioxidant, Anti-Aging and Organ Protective Effects of Total Saponins from Aralia taibaiensis
}

\author{
Huan Li (iD) ${ }^{1,2}$ \\ Bingtao Zhai ${ }^{1,2}$ \\ Jing Sun ${ }^{1,2}$ \\ Yu Fan ${ }^{3}$ \\ Junbo Zou ${ }^{1,2}$ \\ Jiangxue Cheng ${ }^{1,2}$ \\ Xiaofei Zhang ${ }^{1,2}$ \\ Yajun Shi, ${ }^{1,2}$ \\ Dongyan Guo iD 1,2 \\ 'State Key Laboratory of Research \& \\ Development of Characteristic Qin \\ Medicine Resources (Cultivation), \\ Shaanxi University of Chinese Medicine, \\ Xi'an, 7I 2046, People's Republic of \\ China; ${ }^{2}$ The Key Laboratory of Basic and \\ New Drug Research of Traditional \\ Chinese Medicine, Shaanxi University of \\ Chinese Medicine, Xi'an, 7/2046, \\ People's Republic of China; ${ }^{3}$ College of \\ Basic Medicine, Shaanxi University of \\ Chinese Medicine, Xi'an, 7I2046, \\ People's Republic of China
}

Correspondence: Dongyan Guo Tel +86-29-38I8-5I80

Email xmc205I080@163.com

\begin{abstract}
Aim: Aralia taibaiensis is a natural medicinal and food plant that is rich in triterpenoid saponins with hypoglycaemic, antioxidant, hepatoprotective, anti-gastric ulcer and antiinflammatory effects. This study has significance in terms of the antioxidant, anti-aging and organ protective effects of Aralia taibaiensis total saponins (TSAT) in D-galactoseinduced aging rats.
\end{abstract}

Methods: The saponin composition of TSAT was determined and quantified by high performance liquid chromatography (HPLC). We consolidated the antioxidant and enzyme inhibitory activities of TSAT in vitro and assessed the effects of TSAT on daily mobility, body weight, behaviour, organ indices, oxidation-related indices and pathological changes in aging rats.

Results: In vitro experiments showed that TSAT had a scavenging effect on 1,1-diphenyl2-picrylhydrazyl (DPPH), 2,2'-azino-bis(3-ethylbenzothiazoline-6-sulfonic acid) (ABTS), tyrosinase, hydroxyl radicals $(\mathrm{HO} \bullet)$ and superoxide radicals $\left(\cdot \mathrm{O}^{2-}\right)$ and was closely related to the dose of TSAT. In vivo experiments showed that after 8 weeks of continuous gavage administration, the rats gradually recovered their body weight, daily activity ability, learning and memory ability and organ index and effectively improved D-gal-induced organ injury. Specifically, TSAT significantly increased the levels of superoxide dismutase (SOD), catalase (CAT), glutathione peroxidase (GSH-Px) and total antioxidant capacity (T-AOC) and significantly decreased malondialdehyde (MDA) levels in the serum, brain, heart, lung, spleen and kidney of aging rats compared to the model group. In addition, TSAT significantly inhibited the D-gal-induced upregulation of hepatic alanine aminotransferase (ALT) and aspartate aminotransferase (AST) levels. The histopathological results showed that TSAT reversed D-galinduced damage to the brain, heart, lung, kidney, liver and spleen to varying degrees.

Conclusion: TSAT is a high-quality natural product with antioxidant and anti-aging properties that can alleviate D-gal-induced aging damage in rats.

Keywords: Aralia taibaiensis total saponins, D-galactose, antioxidant, anti-aging

\section{Introduction}

Since the 21 st century, with the global trend of aging and the improvement of living standards around the world, health problems caused by aging and aging-related diseases have become increasingly prominent. ${ }^{1}$ Therefore, how to maintain the health of elderly people and delay the occurrence of aging and aging-related diseases has become a popular research topic. Aging is an irreversible and complex degenerative physiological process that occurs in all parts of the body, leading to structural and functional deterioration of tissues and organs and increasing the risk of various chronic diseases, such as hypertension, diabetes, neurodegenerative diseases and skin melanosis. ${ }^{2}$ Several reports have shown that the free radical theory of aging, one of the most popular theories, can explain the damage to the 


\section{Graphical Abstract}

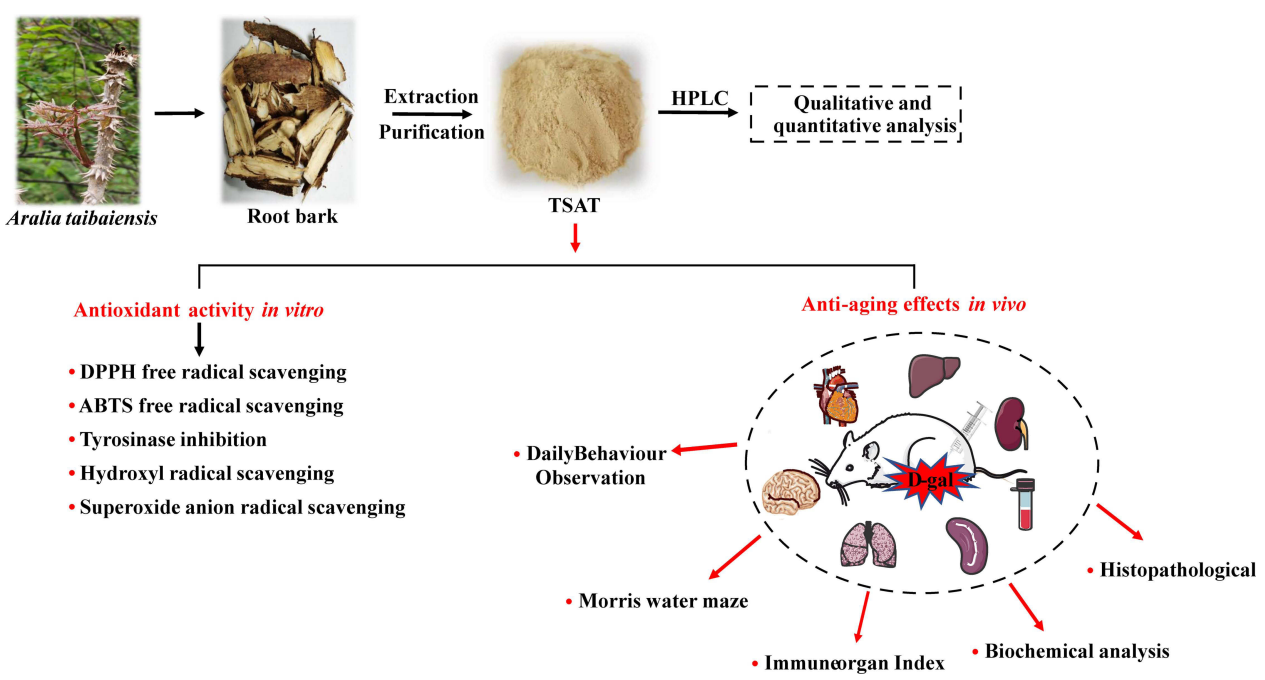

organism caused by the aging process. ${ }^{3}$ During aerobic metabolism, reactive oxygen species (ROS) are produced in cells, including singlet oxygen $\left({ }^{1} \mathrm{O}_{2}\right)$, superoxide anions $\left(\bullet \mathrm{O}^{2-}\right)$, hydroxyl radicals $(\mathrm{HO} \bullet)$, and hydrogen peroxide $\left(\mathrm{H}_{2} \mathrm{O}_{2}\right)^{4,5}$ Once these free radicals, which contain unpaired and highly reactive electrons, are produced in excess, they directly lead to oxidative stress and proinflammatory responses, followed by redox imbalance, leading to protein and lipid peroxidation, as well as mitochondrial and DNA damage, ultimately leading to altered cell and tissue function and pathological conditions to accelerate aging. ${ }^{6,7}$ Interestingly, natural endogenous antioxidant systems exist in the human/mammalian system, including those involving SOD, CAT and GSH-Px, as well as nonenzymatic antioxidants such as ascorbic acid and carotenoids, designed to maintain a proper balance between free radicals and antioxidants. ${ }^{8,9}$ Nevertheless, the antioxidant defence system is not sufficient to completely prevent oxidative damage caused by excess free radicals, and supplementation with antioxidants can at least reduce the extent of aging and the related oxidative damage. ${ }^{10}$ Compared with natural antioxidants, synthetic antioxidants (eg, BHT and BHA) have been shown to cause endocrine disruption, reproductive disorders and even carcinogenesis in long-term use. ${ }^{11,12}$ Therefore, the search for natural, safe and effective free radical scavengers is of great importance for human health protection.

Aralia taibaiensis is a plant species of the same genus as Aralia elata (Miq.) Seem. ${ }^{13}$ It is widely distributed in the Qinba Mountains of western China and is a medicinal and edible plant. Aralia taibaiensis root bark extract has long been used in China as a folk medicine for the treatment of diabetes, and its main active ingredient is the triterpene saponin. ${ }^{14}$ It has been shown to have antioxidant, antihyperglycaemic and antihyperlipidaemic effects. ${ }^{14-16}$ Many studies have shown that triterpene saponins such as asiaticoside $B$, madecassoside and parkioside A can react with free radicals and prevent further development of free radical chain reactions, thus exerting antioxidant effects. ${ }^{17,18}$ Previous reports described that TSAT exhibited superior DPPH radical scavenging activity to single saponins, as well as good antioxidant capacity against lipid peroxidation in rat liver microsomes, and was shown to have superior antioxidant effects to ginseng, schisandra and other saponins. ${ }^{14,15}$ In addition, TSAT also attenuated D-gal-induced liver damage in aging rats through its antioxidant effect. ${ }^{19}$ Further structure-effect relationship study suggested that the structural differences such as the category and the sequence of the oligosaccharide chain at C-3 in such saponins play an important role in terms of diabetes mellitus related antioxidant and antiglycation activities. $^{20}$

D-gal is a commonly used experimental aging inducer. Increasing amounts of evidence suggest that aging-related phenotypes such as excessive free radical formation, cognitive dysfunction, decreased immune response and organ injury can be observed under long-term D-gal stimulation. $^{21}$ Behaviourally, D-gal administration leads 
to deficits in spatial and recognition memory in rats, as demonstrated by poor performance in terms of difficulties in finding target platforms and reduced number of times crossing target platforms in a Morris water maze experiment. $^{22}$ Therefore, chronic D-gal stimulation has been used to study organismal aging and aging-related memory loss, redox imbalance and organ injury.

On the basis of previous studies on the biological activity of TSAT, there is reason to believe that this medicinal and dietary plant has considerable potential for application in the field of anti-aging. However, there is only one study on the in vitro scavenging of free radicals by this triterpenoid saponin extract, and other physiologically relevant characterizations of free radical scavenging are lacking. Furthermore, knowledge on the protective effects of TSAT against memory loss, redox imbalance and organ injury in a D-gal-induced subacute aging model is limited. Therefore, this study consolidated the antioxidant properties of TSAT in vitro by scavenging $\mathrm{DPPH}, \mathrm{ABTS}, \mathrm{HO} \cdot \bullet^{-\mathrm{O}^{2-}}$ and inhibiting tyrosinase activity. Moreover, behavioural experiments, measurement of oxidative stress parameters and histopathological conditions of major organs in rats were evaluated to provide additional information on TSAT's ability to ameliorate D-gal-induced subacute oxidative and aging damage, consolidating its anticipated pharmacological properties.

\section{Materials and Methods}

The root bark of Aralia taibaiensis was collected from the Qinba Mountains, Shaanxi Province, China, and was botanically identified by Dr. Jitao Wang (Shaanxi University of Chinese Medicine). A voucher specimen (SUCM, No. 20201003) was deposited in the Herbarium of Shaanxi University of Chinese Medicine. Tarasaponin IV ( $\geq 98 \%$ ), araloside C ( $\geq 98 \%)$, stipuleanoside R2 ( $\geq 98 \%)$, pseudoginsenoside RT1 ( $\geq 98 \%)$, araloside A ( $\geq 98 \%)$ and chikusetsu saponin Iva ( $\geq 98 \%$ ) standards were provided by Chenguang Biotechnology Co., Ltd (Baoji, China). DPPH (Sigma-Aldrich, St. Louis, MO, USA), ABTS (Maclin Biochemical Co., Ltd, Shanghai, China), mushroom tyrosinase (Yuan Ye Biotechnology Co., Ltd, Shanghai, China), L-3,4-dihydroxyphenylalanine (LDOPA) and D-gal (Yuan Ye Biotechnology Co., Ltd, Shanghai, China) were purchased. Superoxide anion (Item number: A052-1-1), Hydroxyl free radical (Item number: A018-1-1), BCA (Item number: A045-4-2), SOD (Item number: A001-3-2), GSH-Px (Item number: A005-1-1), CAT (Item number: A007-1-1), MDA (Item number: A003-1-2), T-AOC (Item number: A015-2-1), AST (Item number: C010-2-1) and ALT (Item number: C009-2-1) assay kits were obtained from Nanjing JianCheng Bioengineering Institute (Nanjing, China). All chemicals and other reagents used were analytical grade.

\section{Extraction of TSAT}

Aralia taibaiensis root bark was washed with double distilled water, dried and crushed at $60{ }^{\circ} \mathrm{C}$. Before the experiment, the powder was placed in a cool and dry place. The powder $(3 \mathrm{~kg}$ ) was extracted with $70 \%$ ethanol for three times under reflux. The solutions were combined and the supernatant was obtained by high speed centrifugation and filtration. The supernatant was purified by loading into an HPD300 macroporous resin column and then eluting with $70 \%$ ethanol. The solution which eluted with $70 \%$ ethanol was evaporated to yield a residue (356 g) after solvent removal in vacuo. The yield of TSAT was $11.87 \%(\mathrm{w} / \mathrm{w})$ and was preserved in a refrigerator for further use.

\section{HPLC Analysis of TSAT}

The purified TSAT was analysed by HPLC-DAD (Agilent 1260 series liquid chromatograph, USA), which was equipped with a COSMOSIL 5C18-MS-II chromatographic column $(4.6 \mathrm{ID} \times 250 \mathrm{~mm})$. The mobile phase consisted of acetonitrile (solvent $\mathrm{A}$ ) and $0.1 \%$ phosphoric acid aqueous solution (V/V) (solvent B). The gradient programme was as follows: $0-10 \mathrm{~min}, 5-20 \% \mathrm{~A} ; 10-25 \mathrm{~min}$ 20-28\% A; 25-35 min, 28-33\% A; 35-45 min, 33-38\% A; 45-55 min, 38-46\% A; 55-60 min, 46-60\% A; 60-70 min. 60-75\% A; 70-80 min, 75-45\% A; 80-90 min, 45$5 \%$ A. The operating conditions of the instrument were a temperature of $30^{\circ} \mathrm{C}$ and a flow rate of $0.8 \mathrm{~mL} / \mathrm{min}$. The injection volume of standards and samples was $10 \mu \mathrm{L}$. The detection wavelength is $203 \mathrm{~nm}$. Before injection, all samples were passed through a $0.22 \mu \mathrm{m}$ filter. After matching with the standard, the peak is determined by the retention time. The linear calibration curve of the standard was used for quantification.

\section{Antioxidant Activity in vitro DPPH Assay}

The DPPH assay was slightly modified from a previous report. $^{23}$ A mixture of anhydrous ethanol $(100 \mu \mathrm{L}), \mathrm{DPPH}$ solution $(100 \mu \mathrm{L}, 0.2 \mathrm{mM})$ and sample $(100 \mu \mathrm{L}, 0.01-$ $1 \mathrm{mg} / \mathrm{L})$ was shaken vigorously and kept $\left(25{ }^{\circ} \mathrm{C}\right.$, $30 \mathrm{~min}$ ) in the dark. VC was used as a positive control. 
The absorbance $(517 \mathrm{~nm})$ was measured. The DPPH radical scavenging rate was calculated as follows:

$$
\text { DPPH Scavenging rate }(\%)=\frac{A_{0}-\left(A_{1}-A_{2}\right)}{A_{0}} \times 100
$$

where $\mathrm{A}_{0}$ represents the absorbance of the negative control (DPPH plus absolute ethanol); $\mathrm{A}_{1}$ represents the test system containing the sample (DPPH plus TSAT); and $\mathrm{A}_{2}$ is the absorbance of the blank system (TSAT plus absolute ethanol). The $50 \%$ inhibition concentration value $\left(\mathrm{IC}_{50}\right)$ of the DPPH free radical scavenging rate was calculated, which represents the antioxidant capacity of TSAT. All operations are performed in parallel three times.

\section{ABTS+ Assay}

ABTS was determined using the method of Rafique et al with slight modifications. ${ }^{24}$ First, a series of TSAT (0.01$1 \mathrm{mg} / \mathrm{mL}$ ) solutions were prepared. An ABTS solution of 7 $\mathrm{mM}$ and a $\mathrm{K}_{2} \mathrm{~S}_{2} \mathrm{O}_{8}$ solution of $2.45 \mathrm{mM}$ were prepared. After being mixed in the same volume, ABTS+ was obtained by reacting $(12-16 \mathrm{~h})$ in the dark. The prepared ABTS+ was diluted with double distilled water to an absorbance of $0.7 \pm 0.05$ at $734 \mathrm{~nm}$. Then, the mixture of $180 \mu \mathrm{L}$ diluted ABTS+ solution and $20 \mu \mathrm{L}$ sample were reacted $\left(25^{\circ} \mathrm{C}, 10 \mathrm{~min}\right)$ in the dark. VC was used as the positive control. The absorbance $(734 \mathrm{~nm})$ was measured. The ABTS + scavenging capacity was calculated by the following formula:

$$
\text { ABTS }+ \text { Scavenging rate }(\%)=\frac{A_{0}-\left(A_{1}-A_{2}\right)}{A_{0}} \times 100
$$

where $\mathrm{A}_{0}$ represents the absorbance of the negative control (ABTS+ plus double distilled water); $\mathrm{A}_{1}$ represents the test system containing the sample (ABTS + plus TSAT); and $A_{2}$ is the absorbance of the blank system (TSAT plus double distilled water). The $\mathrm{IC}_{50}$ value of $\mathrm{ABTS}+$ scavenging rate was then calculated. All operations were performed in parallel three times.

\section{Determination of Mushroom Tyrosinase Inhibition Activity}

The tyrosinase inhibitory activity was determined according to a slightly improved version of the method previously reported by Morais et al. ${ }^{25}$ A series of $50 \mu \mathrm{L}$ TSAT $(0.01-1 \mathrm{mg} / \mathrm{mL}$, in $50 \%$ DMSO) solutions were added to the reaction system containing $100 \mu \mathrm{L}$ of PBS buffer $(0.1 \mathrm{M}, \mathrm{pH} 6.8)$ and $50 \mu \mathrm{L}$ tyrosinase $(100 \mathrm{U} / \mathrm{mL})$, respectively, and reacted at $25{ }^{\circ} \mathrm{C}$ for $15 \mathrm{~min}$. Then, $50 \mu \mathrm{L} \mathrm{L} \mathrm{L-DOPA}(3.5 \mathrm{mM})$ solution was added to the reaction system and incubated at $37{ }^{\circ} \mathrm{C}$ for $10 \mathrm{~min}$. The absorbance $(475 \mathrm{~nm})$ was determined. VC was used as the positive control. The inhibition activity of TSAT was calculated by the following formula:

$$
\text { Inhibition } \operatorname{rate}(\%)=\frac{A_{0}-\left(A_{1}-A_{2}\right)}{A_{0}} \times 100
$$

where $\mathrm{A}_{0}$ represents the absorbance of the negative control with $50 \%$ DMSO instead of the sample; the system contains L-DOPA and tyrosinase; $\mathrm{A}_{1}$ represents the test system containing the sample and contains L-DOPA and tyrosinase; and $\mathrm{A}_{2}$ is the blank system (sample plus L-DOPA) absorbance. The $\mathrm{IC}_{50}$ value was calculated to indicate the tyrosinase inhibitory activity of TSAT. All operations were performed in parallel three times.

\section{Hydroxyl Radical (HO•) Scavenging Assay}

The Fenton reaction is the most common chemical reaction that produces hydroxyl radicals. This experiment was carried out according to the instructions of the hydroxyl radical assay kit. Different concentrations of TSAT (0.01$1 \mathrm{mg} / \mathrm{mL}$ ) were mixed with the reagents sequentially and left at room temperature for $20 \mathrm{~min}$. Absorbance $(550 \mathrm{~nm})$ was measured, and VC was used as a positive control. The scavenging rate (\%) was calculated as follows:

$$
\mathrm{HO} \bullet \text { Scavenging } \operatorname{rate}(\%)=\frac{A_{0}-\left(A_{1}-A_{2}\right)}{A_{0}} \times 100
$$

where $A_{0}$ is the absorbance of the negative control system without TSAT, $A_{1}$ is the absorbance of the reaction system containing TSAT, and $\mathrm{A}_{2}$ is the absorbance of the reaction system containing double distilled water instead of $\mathrm{H}_{2} \mathrm{O}_{2}$. The $\mathrm{IC}_{50}$ value was then calculated. All operations were performed in parallel three times.

\section{Superoxide Radical $\left(\cdot \mathrm{O}^{2-}\right)$ Scavenging Assay}

The inhibition of superoxide anion radical kits has biological significance by simulating the reaction system of xanthine and xanthine oxidase to generate superoxide anion radicals. In this experiment, the reagents were prepared according to the kit instructions, different concentrations of TSAT solution $(0.01-1 \mathrm{mg} / \mathrm{mL})$ were added, and a water bath at $37^{\circ} \mathrm{C}$ for $40 \mathrm{~min}$. VC was used as a positive control. The scavenging rate (\%) was calculated as follows:

$$
\bullet \mathrm{O}^{2-} \text { Scavenging rate }(\%)=\frac{A_{0}-\left(A_{1}-A_{2}\right)}{A_{0}} \times 100
$$

where $\mathrm{A}_{0}$ represents the absorbance of the negative control with deionized water instead of the sample; $A_{1}$ represents 
the test system containing the sample; and $\mathrm{A}_{2}$ is the absorbance of the blank system. The $\mathrm{IC}_{50}$ value was then calculated. All operations were performed in parallel three times.

\section{Antioxidant Activity in vivo Animals and Treatment}

Male Sprague-Dawley rats (200-230 g) were supplied by the Chengdu Dossy Experimental Animals co., Ltd (ChengDu, China, certificate no. SCXK2020-030). All animal experiments were performed in accordance with the National Institutes of Health Guide for the Care and Use of Laboratory Animals and were approved by the Institutional Animal Ethics Committee of Shaanxi University of Chinese Medicine. These rats were raised in a climate control room $\left(24-25{ }^{\circ} \mathrm{C}\right)$ according to a $12 \mathrm{~h}$ light/12 $\mathrm{h}$ dark schedule and had free access to food and water. All rats were allowed to adapt for a week before starting the experiment. The rats were randomly divided into 6 groups $(\mathrm{n}=8$ in each group): normal group; model group; VC treatment group $(100 \mathrm{mg} / \mathrm{kg})$; and TSAT high (TSAT-H)-, medium (TSAT-M)- and low (TSAT-L)dose treatment groups (200 mg/kg, $100 \mathrm{mg} / \mathrm{kg}, 50 \mathrm{mg} / \mathrm{kg}$ ). All five groups were injected intraperitoneally with D-gal (200 $\mathrm{mg} / \mathrm{kg}$ ) except for the normal group, which was treated with $0.9 \% \mathrm{NaCl}(10 \%, \mathrm{w} / \mathrm{v})$. The rats in the treatment group were given the drug by gavage once daily, and the dose was continuously adjusted according to body weight for 8 weeks.

After the last administration, the rats fasted for one day. Blood samples were collected immediately after anaesthesia in all rats, and serum was collected by centrifugation $\left(3500 \mathrm{rpm}, 15 \mathrm{~min}, 25^{\circ} \mathrm{C}\right)$. The thymus, heart, liver, spleen, lung, kidney, and testicular tissues were separated quickly, rinsed with ice $0.9 \% \mathrm{NaCl}$ solution, and weighed accurately for the following analysis.

\section{Morris Water Maze (MWM) Test}

The Morris water maze (MWM) experiment was performed according to a previous description with slight modifications. ${ }^{26}$ Briefly, the rats were trained for five running days ( 6 trials/per day) to find the escaped platform, which was hidden $1 \mathrm{~cm}$ in water (water temperature, 25 $\pm 1{ }^{\circ} \mathrm{C}$ ) using a fixed array of cues outside the swimming pool (diameter $160 \mathrm{~cm}$, height $60 \mathrm{~cm}$ ). The swimming pool was divided into four equal quadrants. The arrowhead cues around the pool were kept in the same location throughout the whole training and testing time. After a five-day training, each mouse was allowed to swim for $120 \mathrm{~s}$. After finding the platform, rats were allowed to stay there for $15 \mathrm{~s}$. Beyond that, the rat that failed to locate the platform within the stipulated period (120 s) was placed on it for $15 \mathrm{~s}$. The escape latencies of these rats were recorded as $120 \mathrm{~s}$. Later, the platform was removed, and the rats were placed in the quadrant, which was contrary to the target quadrant, and were allowed to swim freely for $120 \mathrm{~s}$. The numbers of platform quadrant crossings were recorded in the target quadrant. The data were analysed by an automated tracking system.

\section{Biochemical Analysis}

The brain, lung, liver, spleen, heart and kidney tissues were homogenized quickly in ice-cold $0.9 \% \mathrm{NaCl}$ solution $(10 \%$, $\mathrm{w} / \mathrm{v}$ ). After centrifugation (3500 rpm, $15 \mathrm{~min}, 25^{\circ} \mathrm{C}$ ), the supernatant was collected for biochemical analysis. Using bovine serum albumin as the standard, the tissue protein concentration was determined. The levels of SOD, GSH-Px, CAT, MDA, and T-AOC in rat serum and brain, heart, lung, spleen and kidney homogenates were determined according to the standard procedures of the kit, and the liver ALT and AST levels were determined according to the kit instructions.

\section{Histopathological Analysis}

The brain, heart, lung, kidney, liver and spleen tissues were quickly removed and fixed in $4 \%$ paraformaldehyde. $24 \mathrm{~h}$ later, paraffin embedding was performed. Sections were sectioned longitudinally to $4 \mu \mathrm{m}$ thickness, hydrated and stained with haematoxylin-eosin (H\&E). Histomorphology was observed microscopically.

\section{Statistical Analysis}

The results of the behaviour tests, organ index and enzyme activities are expressed as the means \pm SD. The data were analysed by one-way analysis of variance (ANOVA) followed by Tukey's test for post hoc analysis. For novel object recognition, statistical evaluation was performed by a paired sample test. Statistical significance was set at $\mathrm{p}<005$.

\section{Results}

\section{Analysis of TSAT Composition}

As shown in Figure 1, HPLC analysis revealed that TSAT contained six types of saponins, including tarasaponin IV, araloside C, stipuleanoside R2, pseudoginsenoside RT1, araloside $\mathrm{A}$ and chikusetsu saponin Iva. Table 1 shows the content of six saponins in TSAT. 

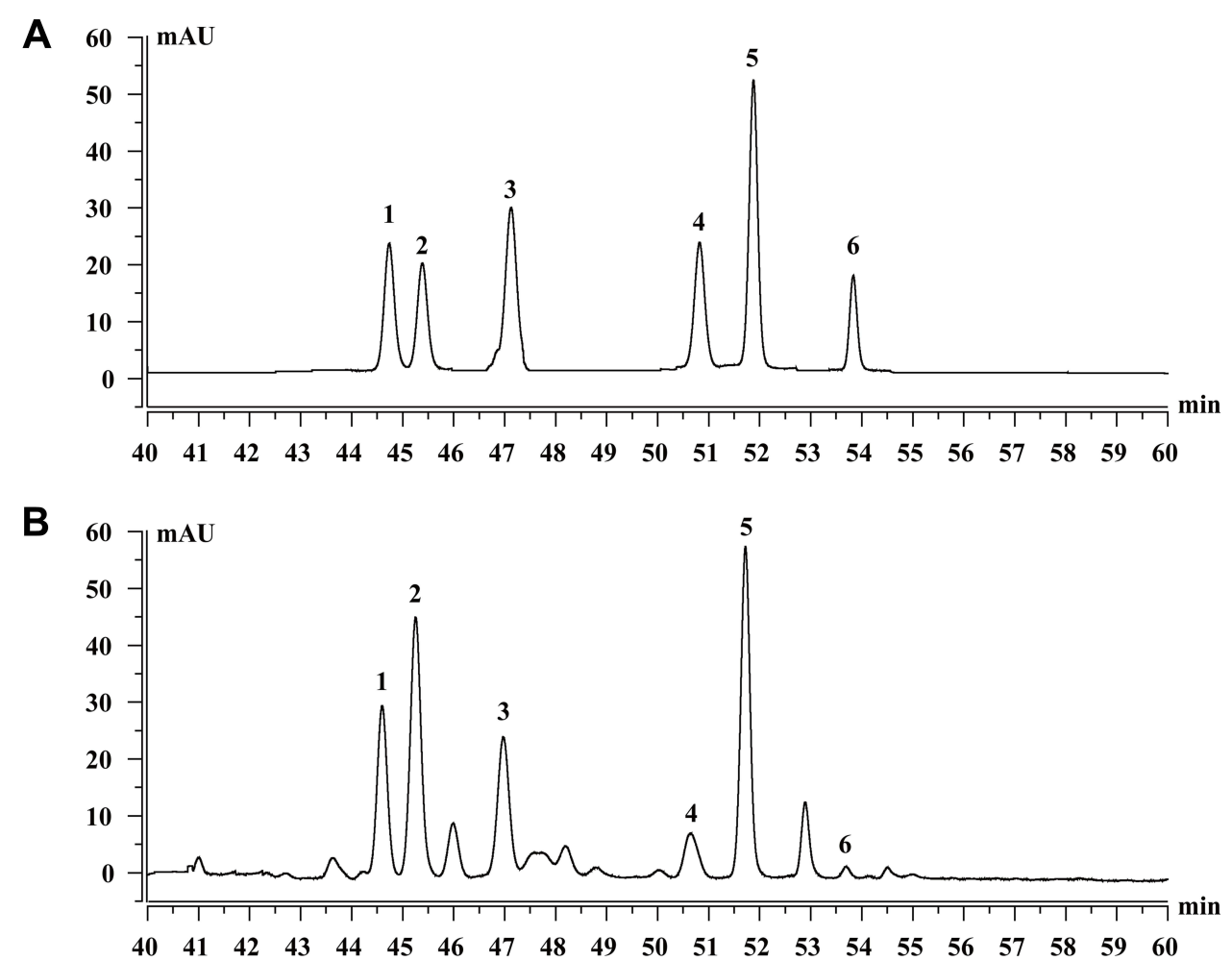

Figure I Saponins constituents of TSAT. (A) Standard chromatograms; (B) Saponins of TSAT chromatograms. I: Tarasaponin IV; 2: Araloside C; 3: Stipuleanoside R2; 4: Pseudoginsenoside RTI; 5: Araloside A; 6: Chikusetsu saponin IVa.

\section{Antioxidant Capacities in vitro}

As shown in Figure 2A, TSAT provided single electrons for pairing with DPPH radicals, and as the concentration of TSAT increased $(0.01-1 \mathrm{mg} / \mathrm{mL})$, the colour of the reaction system gradually became lighter, obeying a significant dose-dependent relationship. The reaction system was almost colourless when TSAT concentrations reached $1 \mathrm{mg} / \mathrm{mL}$, reaching a scavenging rate of $90.5 \pm$ $1.4 \%$, close to that of $\mathrm{VC}$. In addition, the $\mathrm{IC}_{50}$ values of DPPH radical scavenging by TSAT and VC were $0.27 \mathrm{mg} /$ $\mathrm{mL}$ and $5.19 \mu \mathrm{g} / \mathrm{mL}$, respectively. The results indicate that TSAT has the ability to scavenge DPPH free radicals.

The scavenging effect of TSAT on ABTS free radicals is shown in Figure 2B. When the concentration increased

Table I Contents of TSAT Saponins

\begin{tabular}{|l|c|c|}
\hline No. & Component & Content (mg/g) \\
\hline 1 & Tarasaponin IV & 87.13 \\
2 & Araloside C & 165.69 \\
3 & Stipuleanoside R2 & 105.58 \\
4 & Pseudoginsenoside RTI & 41.66 \\
5 & Araloside A & 173.87 \\
6 & Chikusetsu saponin Iva & 12.61 \\
\hline
\end{tabular}

from 0.01 to $1 \mathrm{mg} / \mathrm{mL}$, the scavenging rate increased from $1.56 \pm 3.46 \%$ to $56.01 \pm 3.40 \%$. It was found that the scavenging effect of TSAT on ABTS free radicals was dose-dependent. The $\mathrm{IC}_{50}$ value was $0.78 \mathrm{mg} / \mathrm{mL}$. This shows that increasing the concentration of TSAT can significantly improve the scavenging ability of ABTS free radicals. Under the same conditions, the $\mathrm{IC}_{50}$ value of $\mathrm{VC}$ scavenging ABTS free radicals was $15.48 \mu \mathrm{g} / \mathrm{mL}$.

The tyrosinase inhibitory activity of TSAT was evaluated with L-DOPA as a substrate in vitro. As shown in Figure $2 \mathrm{C}$, the inhibition rate of tyrosinase activity by TSAT obeyed a dose dependence at concentrations of $0.01-1 \mathrm{mg} / \mathrm{mL}$. The inhibition rate of tyrosinase activity by TSAT was $48.55 \pm 2.62 \%$, and the $\mathrm{IC}_{50}$ value was $1.18 \mathrm{mg} / \mathrm{mL}$ at $1 \mathrm{mg} / \mathrm{mL}$. TSAT was proven to be a potential tyrosinase activity inhibitor in the present study. In this study, it was proven to be a potential tyrosinase activity inhibitor for the first time; thus, it may have a potential whitening effect.

Figure 2D shows that the clearance of $\mathrm{HO} \bullet$ by TSAT was only $3.04 \pm 1.93 \%$ at a concentration of $0.01 \mathrm{mg} / \mathrm{mL}$ and reached $56.86 \pm 1.31 \%$ at $1 \mathrm{mg} / \mathrm{mL}$ with increasing TSAT concentration. The $\mathrm{IC}_{50}$ value was $0.528 \mathrm{mg} / \mathrm{mL}$. In conclusion, TSAT has a good scavenging capacity for 

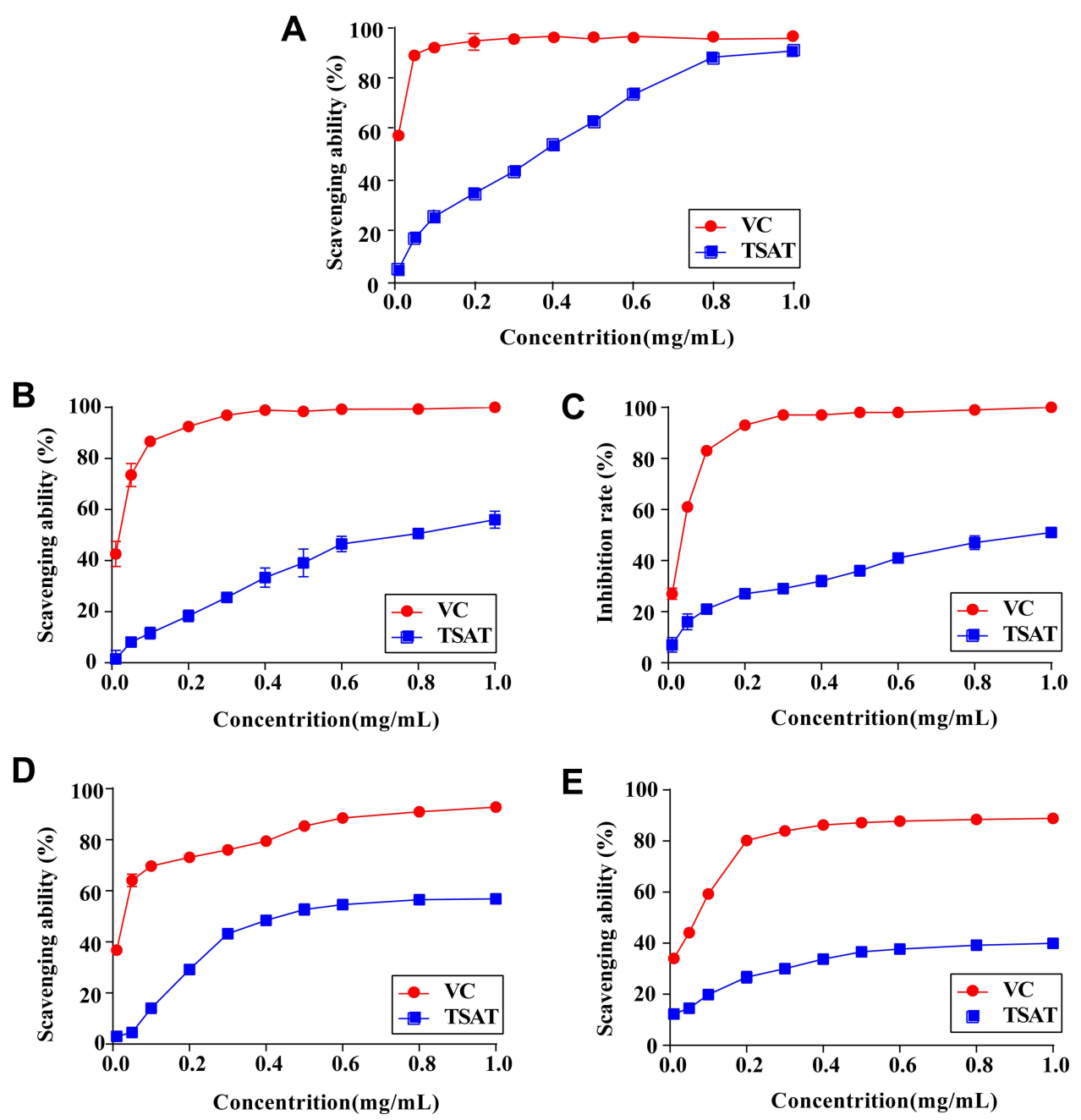

Figure 2 Antioxidant activities of TSAT in vitro. (A) DPPH radicals, (B) ABTS radicals, (C) Tyrosinase, (D) Hydroxyl radicals and (E) Superoxide anion radicals. Abbreviation: VC, Vitamin C.

HO•. It is speculated that TSAT may act as a metal ion chelating agent to block the formation of $\mathrm{HO}$.

In this study, the scavenging rate of $\bullet \mathrm{O}^{2-}$ by TSAT was $39.94 \pm 0.49 \%$ at a concentration of $1 \mathrm{mg} / \mathrm{mL}$, with an $\mathrm{IC}_{50}$ value of $2.43 \mathrm{mg} / \mathrm{mL}$. In addition, Figure $2 \mathrm{E}$ shows that the scavenging rate in $\cdot \mathrm{O}^{2-}$ quenching correlated with the concentration of TSAT.

\section{Anti-Aging Activities Assaying Daily Behaviour Observations and Organ Indices}

During the eight weeks of our experiment, the daily appearance and behavioural activities of the rats were observed and recorded weekly. The rats in the normal group were not only in good mental condition but were also lively, active and agile and had a healthy diet. As shown in Figure 3A, after the 5th week of D-gal injection, the rats in the model group showed a decline in body weight accompanied by obvious signs of senescence, including slow movement, gradually reduced food intake, depression and dry fur, indicating that the senescence model triggered by intraperitoneal injection of D-gal had been successfully established. The above senescence features were regressed to different degrees in both the $\mathrm{VC}$ group and the treated rats compared with the model group. In particular, in the high-dose TSATtreated group, the abovementioned aging characteristics of the rats were significantly reduced compared with those of the model group. Aging causes degenerative atrophy of organs, leading to a decrease in organ indices. ${ }^{27,28}$ In this study, thymus, liver, spleen, kidney and heart organ indices were highest in normal control rats and lowest in the model group $(\mathrm{p}<0.05)$ (Figure 3 ), indicating that D-gal caused organ atrophy in rats. Notably, the TSAT-induced increase in organ indices may reflect the prevention of atrophy of body tissues. 

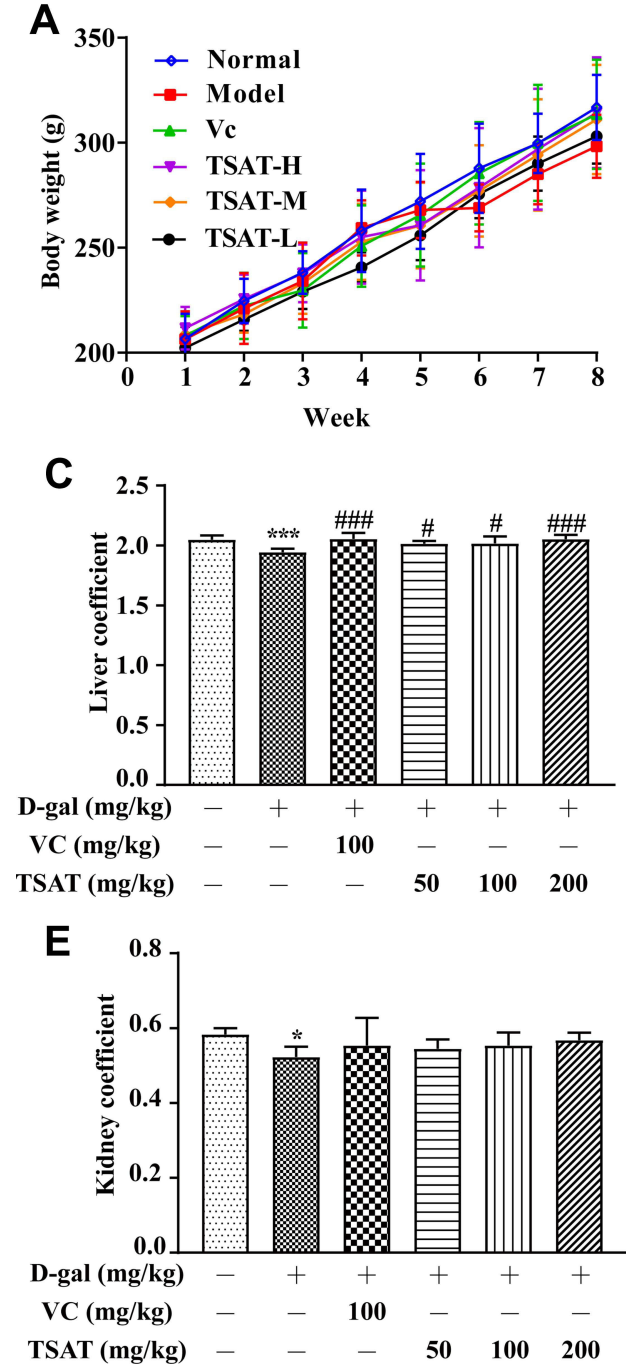
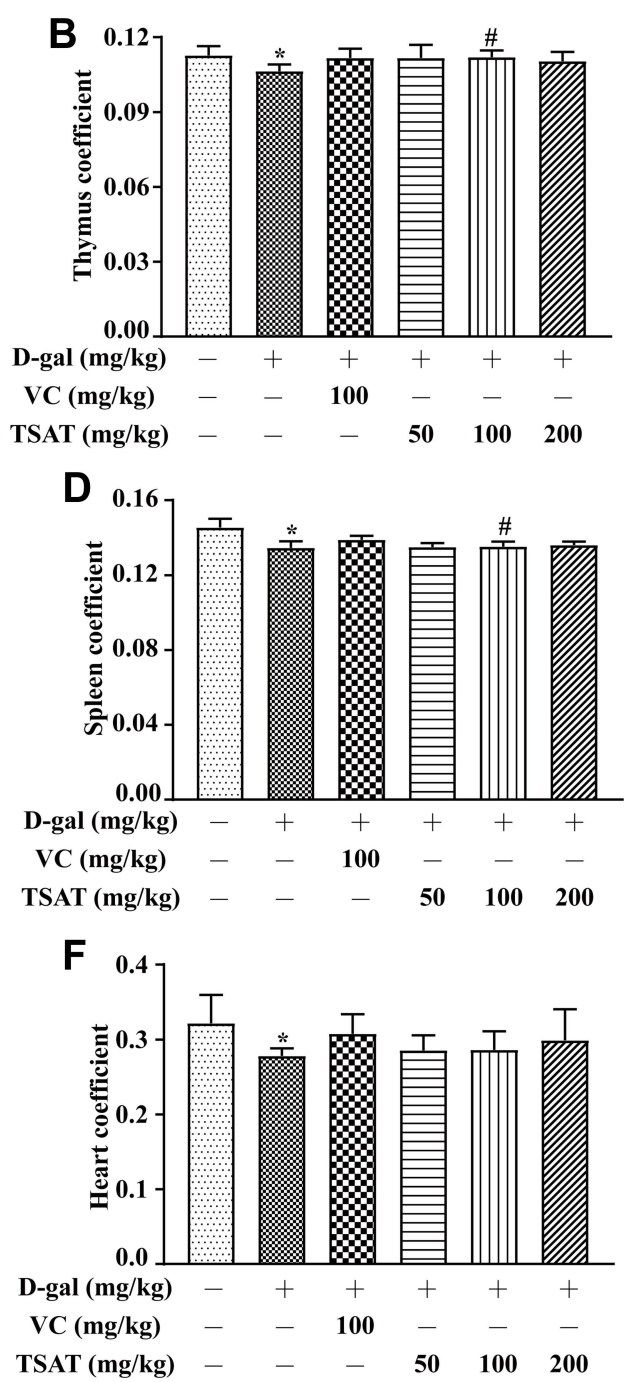

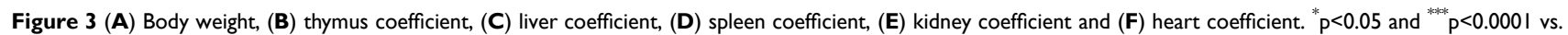
normal group; ${ }^{*}<<0.05$ and ${ }^{\# \#} p<0.0001$ vs. model group. The data are presented as the means \pm SD $(n=8)$.

\section{Morris Water Maze (MWM) Test}

We used the MWM test to assess the effects of TSAT on spatial learning and memory abilities in a D-gal-induced mouse model. As shown in Figure 4A and B, rats in the D-gal group suffered a significant impairment in spatial learning ability during the 5-day location navigation training, as evidenced by a longer escape latency compared to control rats, whereas rats treated with TSAT had a significantly shorter escape latency, which was closer to the normal level. To assess spatial memory more directly, rats were subjected to another test in which the target platform was removed the day after navigation training. As shown in Figure 4C, the rats given D-gal crossed the location less often than the control group. However, compared to those in the model group, the rats in the treatment group had less time to escape the latency and crossed the platform more often, with the higher dose group being more significant $(\mathrm{p}<0.05)$. These results suggest that the aging model impaired spatial learning and memory, while oral administration of TSAT restored age-related cognitive deficits induced by D-gal exposure.

\section{Oxidation-Related Index Assays}

SOD levels in serum, brain, heart, lung, spleen and kidney were significantly lower in the untreated model group than in the normal group $(\mathrm{p}<0.01)$ (Figure 5), indicating that the organism was damaged by oxidative stress to different 

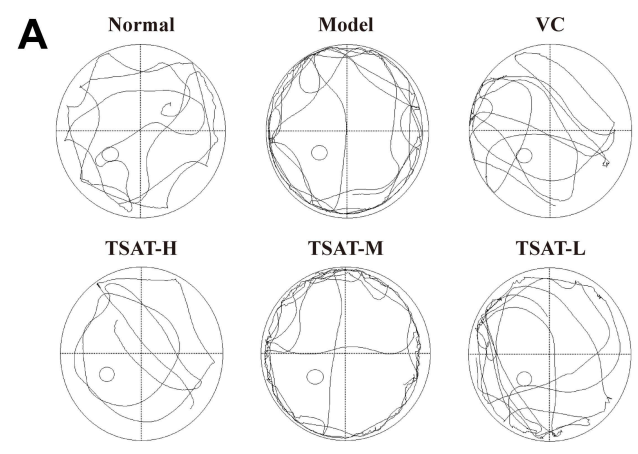

Day 1
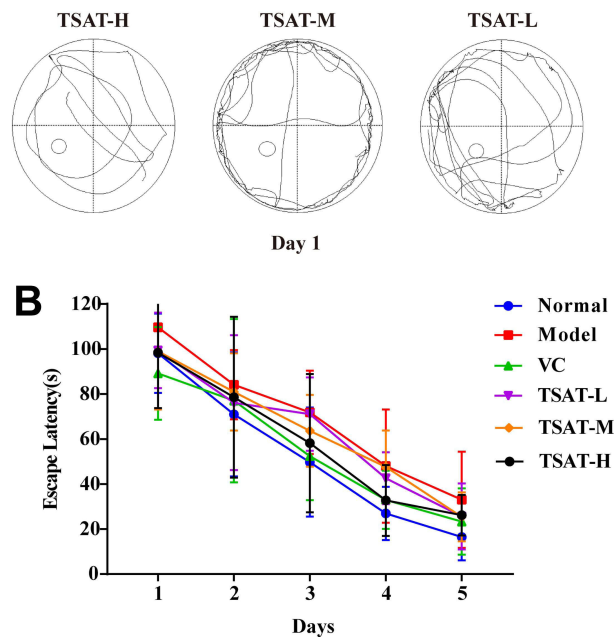
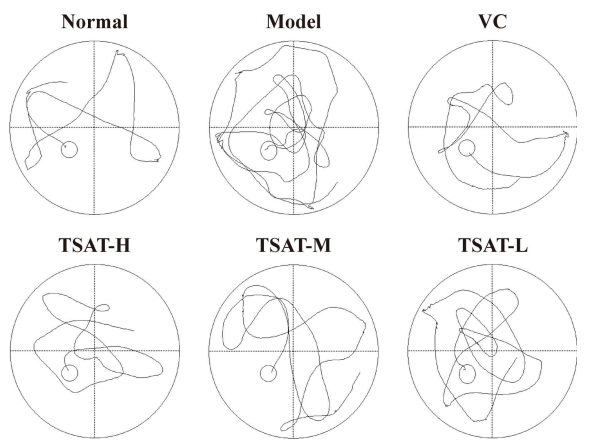

Day 5

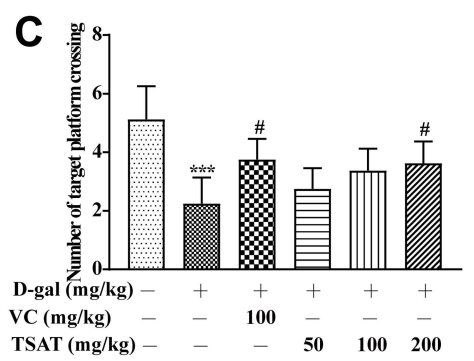

Figure 4 Morris water maze test. (A) Representative pictures of swimming traces on the Ist day and 5th day in the D-gal model; (B) TSAT treatment reduced escape latency time in the D-gal model. (C) TSAT treatment increased across platform times in the D-gal model. ${ }^{* * *} \mathrm{p}<0.000 \mathrm{I}$ vs. normal group; ${ }^{*} \mathrm{p}<0.05$ vs. model group. The data are presented as the means $\pm S D(n=8)$.

degrees. However, the SOD levels in the aging rats administered different doses of TSAT were significantly higher than those in the model group $(\mathrm{p}<0.05)$. The results indicated that TSAT could reverse the decrease in SOD content in aging tissues and serum, with the best effect in the high-dose group.

Compared with the MDA value of normal rats, the MDA levels in the serum, brain, heart, lung, spleen and kidney of the untreated model group were significantly increased $(p<0.01)$ (Figure 6), indicating that organs suffer from oxidative stress injury. In contrast, TSAT treatment downregulated MDA accumulation $(\mathrm{p}<0.05)$. The results indicate that the potential anti-aging properties of TSAT may be related to its reduction in MDA content.

The levels of CAT were significantly lower $(\mathrm{p}<0.01)$ in the serum, brain, heart, lung, spleen and kidney of rats in the untreated model group than in the normal group (Figure 7), indicating that the accumulation of $\mathrm{H}_{2} \mathrm{O}_{2}$ in the organism led to structural cell damage. However, CAT levels improved after treatment in both the VC and TSAT administration groups $(\mathrm{p}<0.05)$, especially in the serum and organ CAT levels of rats in the high-dose TSAT group, which were significantly higher than those in the model group.
GSH-Px was significantly lower $(\mathrm{p}<0.01)$ in the serum, brain, heart, lung, spleen and kidney of untreated aging rats (Figure 8) than in the normal group of rats. In contrast, GSH-Px activity was significantly increased after TSAT administration, with a significant difference compared to that in the model group $(\mathrm{p}<0.05)$, possibly acting by blocking free radical damage to cell membrane lipids.

As shown in Figure 9, serum, brain, heart, lung, spleen and kidney T-AOC levels were significantly lower in the untreated aging group than in the normal group $(\mathrm{p}<0.01)$. However, TSAT treatment significantly increased T-AOC levels $(p<0.05)$. This may be due to the long-term consumption of antioxidant enzymes caused by high levels of oxidative stress, which was reversed by oral TSAT. This result strongly indicates that TSAT has antioxidant potential and can increase antioxidant capacity, thus reducing the damage caused by aging.

ALT and AST are effective markers for evaluating liver function injury. ${ }^{29}$ As shown in Figure 10, compared with those in the normal group of rats, the levels of ALT and AST in the untreated model group were significantly increased $(\mathrm{p}<0.01)$, indicating that the liver was damaged by D-gal stimulation. In contrast, after administration of TSAT, the levels of ALT and AST in the livers of aging 

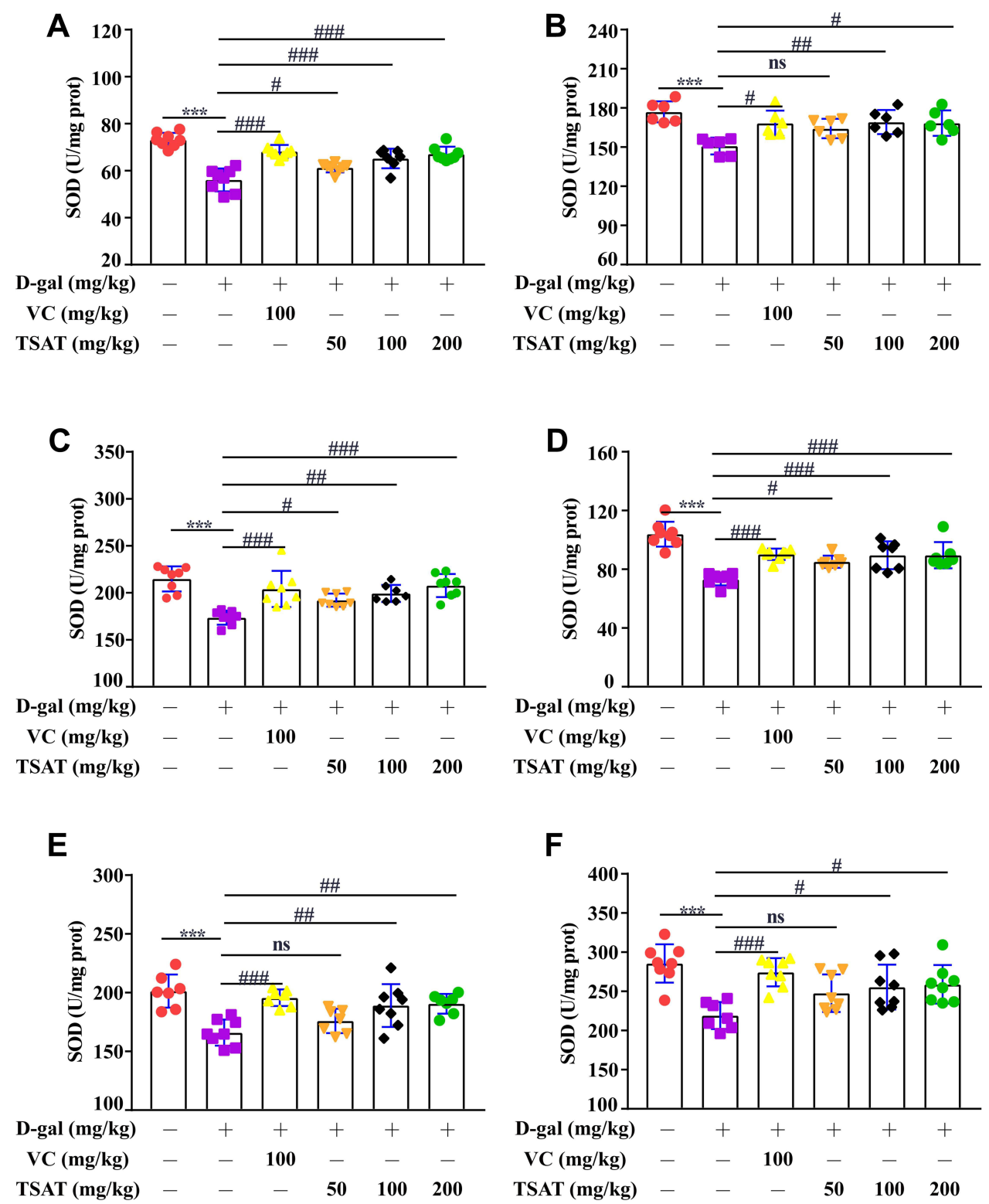

Figure 5 Effect of TSAT on SOD levels in (A) serum, (B) brain, (C) heart, (D) lung, (E) spleen and (F) kidney. ${ }^{* * *}$ p $<0.000$ I vs normal group; ${ }^{\#}<<0.05$, ${ }^{\#}{ }_{p}<0.0$ I and \# $<0.000$ I vs model group. All the data are expressed as the means \pm SD of 6-8 rats in each group.

rats were significantly reduced $(\mathrm{p}<0.05)$. The results show that TAST has a protective effect on the liver.

\section{Histopathological Observations}

In this study, histopathological observations were performed on the brain, heart, lung, kidney, liver and spleen to confirm the evidence from the biochemical analysis. Figure 11 shows the effect of TSAT on the histopathology of D-gal-induced aging rats. It is obvious that organ injury is mainly manifested by the degradation of cells and nuclei. In addition, senescent rats exhibited other pathological patterns in these organs, such as partial edema, fragmented arrangement of nerve cells and atrophy of nuclei in brain tissue (Figure 11A), broken, loosely arranged and increased intercellular fibres in the myocardium (Figure 11B), alveolar haemorrhage, thickened alveolar walls and inflammatory cell infiltration in the alveolar space and around blood vessels (Figure 11C), glomerular atrophy and possible proximal tubules of the kidney balloon dilatation lumen (Figure 11D), binucleation of hepatocytes, hepatic cord disorder and swelling of hepatocytes (Figure 11E), and old haematopoiesis in the spleen with increased fibrous septa and fused lymphoid follicles (Figure 11F). Interestingly, TSAT treatment 

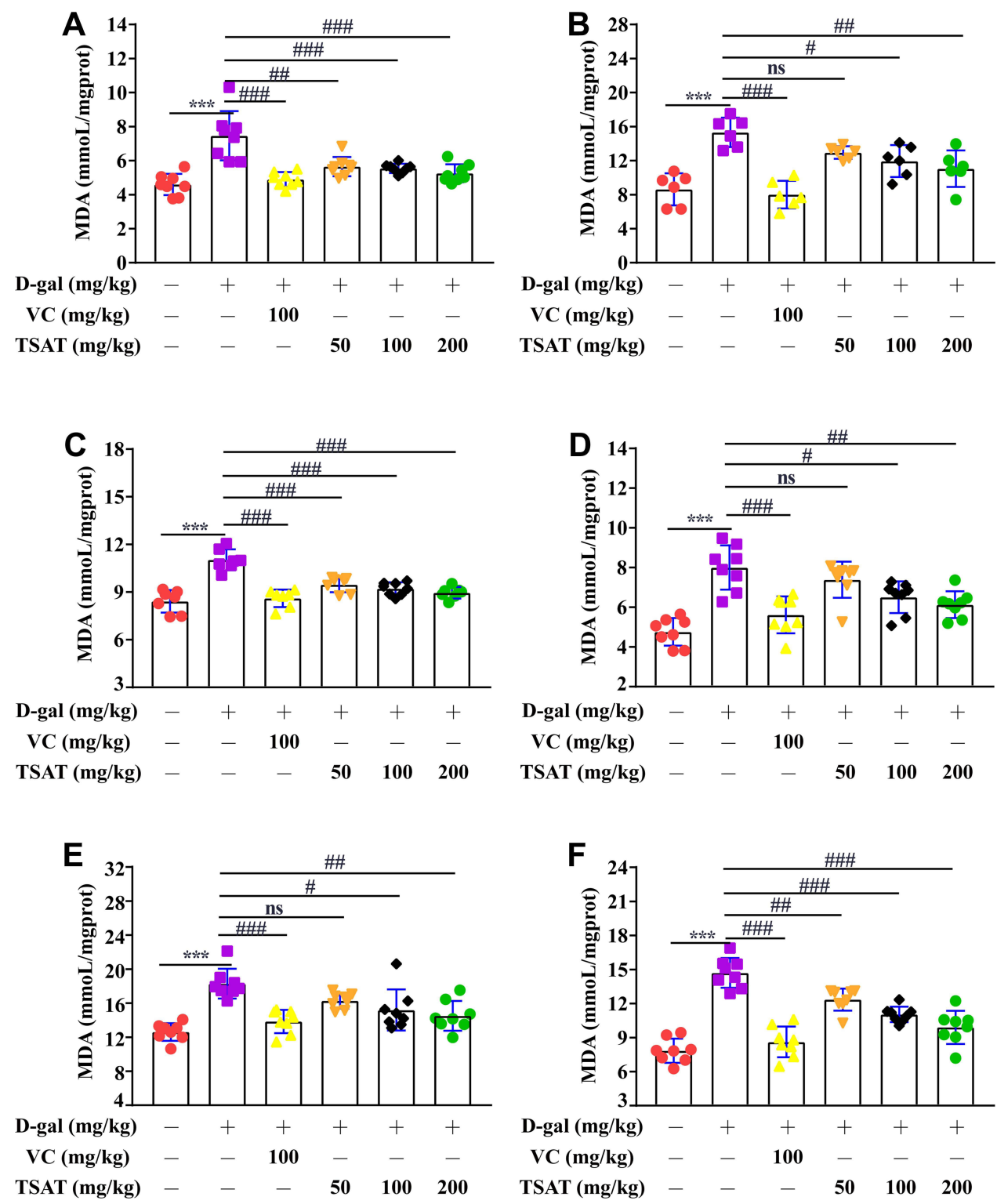

Figure 6 Effect of TSAT on MDA levels in (A) Serum, (B) Brain, (C) Heart, (D) Lung, (E) Spleen and (F) Kidney. ${ }^{* * *} \mathrm{p}<0.000 \mathrm{I}$ vs normal group; ${ }^{\#}<<0.05$, ${ }^{\# \#}$ p $<0.0$ I and $\mathrm{p}<0.000$ I vs model group. All the data are expressed as the means \pm SD of 6-8 rats in each group.

significantly attenuated the adverse organ lesions caused by D-gal, with the best results seen in the high-dose group. Moreover, the vitamin C-treated control rats had a similar recovery effect. The results suggest that TSAT has potential protective effects on the brain, heart, lung, kidney, liver and spleen against acute D-gal-induced injury.

\section{Discussion}

In recent years, TSAT has been shown to have high antioxidant activity and contribute to the prevention of ROSinduced oxidative damage in the body. Based on the free radical theory of aging, long-term injection of D-gal causes oxidative stress by stimulating excessive production of ROS, leading to metabolic disorders and inducing oxidative damage to organs in vivo associated with aging. $^{30,31}$ Therefore, D-gal-induced aging models have been widely used in anti-aging and organ injury studies, for which antioxidant supplementation may be a potential therapeutic strategy for D-gal-induced oxidative and aging damage. In this study, the antioxidant activity of TSAT was consolidated by scavenging DPPH, ABTS, hydroxyl radicals and superoxide anion radicals and inhibiting tyrosinase activity in vitro. Furthermore, behavioural evaluation, organ indices, biological indicators of oxidative stress and analysis of pathological changes revealed that D-gal-induced aging rats had 

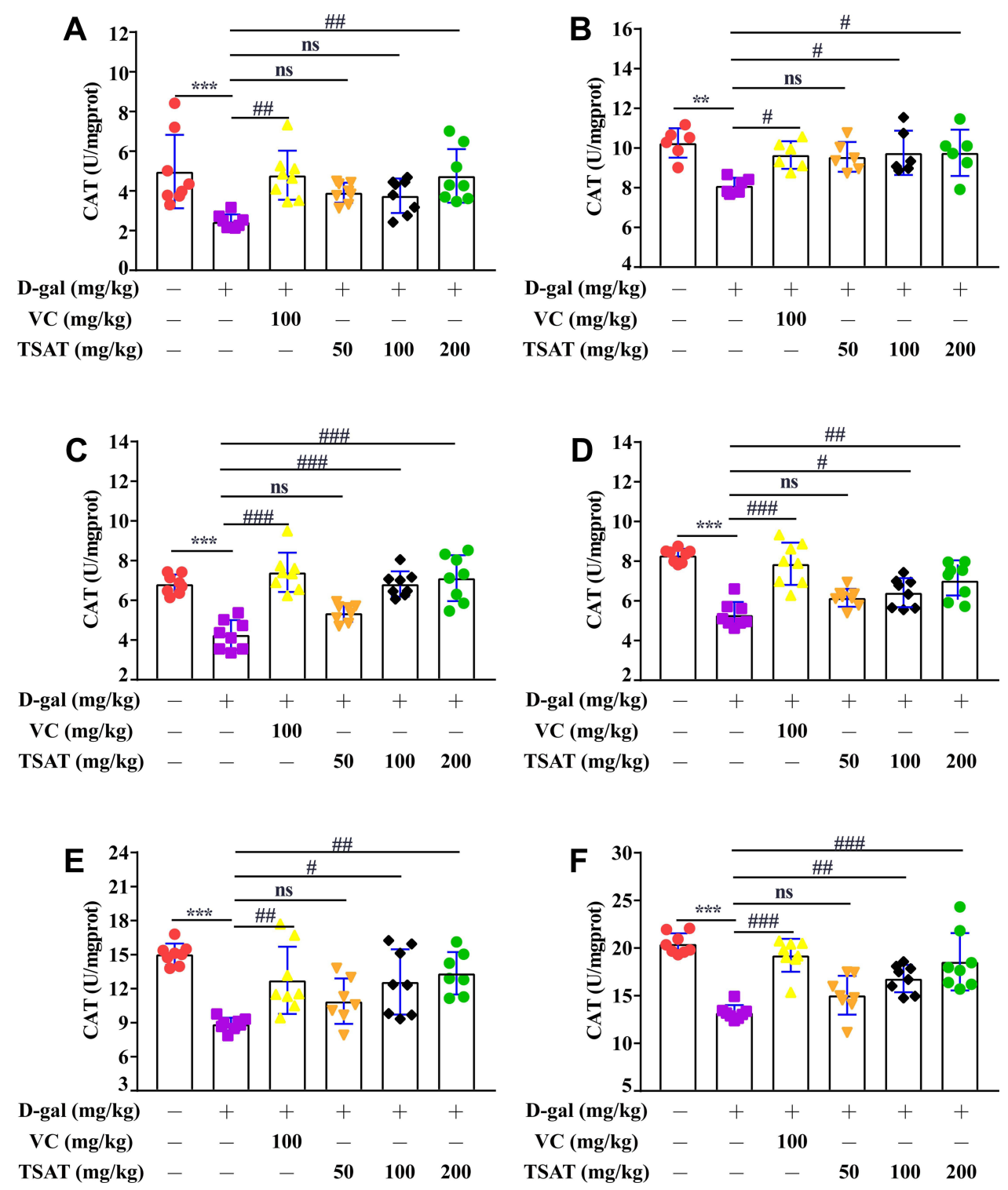

Figure 7 Effect of TSAT on CAT levels in (A) Serum, (B) Brain, (C) Heart, (D) Lung, (E) Spleen and (F) Kidney. ${ }^{* *} \mathrm{p}<0.001$, ${ }^{* * *} \mathrm{p}<0.000$ I vs normal group; ${ }^{\mathrm{p}}<0.05$, ${ }^{*} \mathrm{p}<0.01$ and ${ }^{\# \#}<0.0001$ vs model group. All the data are expressed as the means \pm SD of 6-8 rats in each group.

reduced major organ indices and reduced learning memory capacity with varying degrees of oxidative damage, but this effect was inhibited by TSAT intervention.

HPLC was used to analyse TSAT in this study, and the results showed that TSAT contained six triterpene saponins, including araloside $\mathrm{C}$, Chikusetsu saponin IVa, araloside A, pseudoinsenoside RT1, tarrasaponin IV and stipuleanoside R2. Araloside $\mathrm{C}$ and Chikusetsu saponin IVa have been shown to have antioxidant properties, reduce the accumulation of ROS in cells, inhibit apoptosis and reduce $\mathrm{Ca}^{2+}$ overload. ${ }^{32,33}$ It has been reported that mitochondrial dysfunction generates excess reactive oxygen species and contributes to aging-related diseases. ${ }^{34}$
Araloside $\mathrm{C}$ reduces mitochondrial ROS levels by increasing cell viability and improving disruption of mitochondrial membrane potential. ${ }^{35}$ However, whether other saponins have similar effects need to be further investigated. These results suggest that the anti-aging effect of TSAT is related to the scavenging effect on ROS associated with its active saponins.

Increasing evidence has shown that ROS such as superoxide anion, hydrogen peroxide and hydroxyl free radicals can attack life macromolecules and cell walls, thus inducing oxidative stress, causing various oxidative damage and pathological changes, and accelerating aging. ${ }^{10,36,37}$ The present work evaluates the antioxidant activity of 

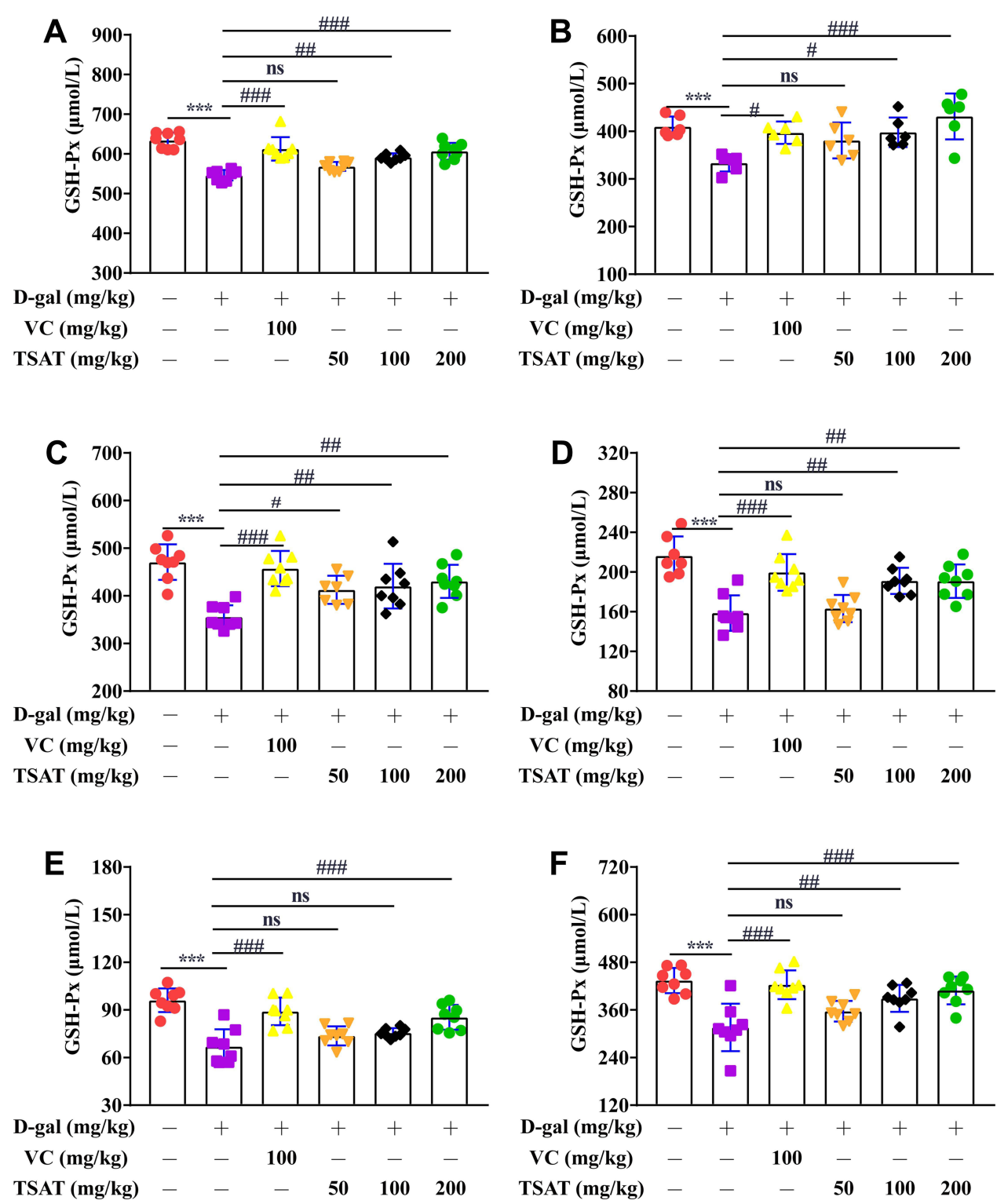

Figure 8 Effect of TSAT on GSH-PX levels in (A) Serum, (B) Brain, (C) Heart, (D) Lung, (E) Spleen and (F) Kidney. ${ }^{* * *}$ p $<0.000$ I vs normal group; ${ }^{*}<<0.05$, ${ }^{\#}$ p $<0.0$ I and P $<0.000$ I vs model group. All the data are expressed as the means \pm SD of $6-8$ rats in each group.

TSAT by measuring the scavenging of DPPH radicals, ABTS radicals, tyrosinase, hydroxyl radicals and superoxide anions in vitro. Among them, DPPH is one of the stable and mature organic nitrogen free radicals and has the maximum absorption at $517 \mathrm{~nm}$. After adding antioxidants, electrons or hydrogen atoms are transferred to DPPH to form stable DPPH-H compounds, which reduces its absorption at $517 \mathrm{~nm} .{ }^{38}$ In ABTS analysis, ABTS was converted to ABTS+ by adding $\mathrm{K}_{2} \mathrm{~S}_{2} \mathrm{O}_{8}$. In the presence of antioxidants, active ABTS+ was converted into a colourless natural form. Tyrosinase plays a key role in melanin formation and browning. Under ultraviolet radiation stimulation, the excessive production and accumulation of melanin in the skin may lead to pigmentation disorders and skin aging, and the down-regulation of tyrosinase is considered to be the reason for the decrease in melanin activity. Tyrosinase has also been reported to be associated with neurodegenerative diseases. When produced in excess, tyrosinase is able to increase intracellular dopamine production, followed by the induction of melanin formation, causing cell death. ${ }^{39,40}$ Interestingly, some studies have proved that saponins could be potential natural tyrosinase inhibitors. For example, saponins from Xanthoceras Sorbifolia nutshell were reported to inhibit $52.0 \%$ of tyrosinase activity at a concentration of $0.96 \mathrm{mg} /$ $\mathrm{mL} .{ }^{41}$ Furthermore, some transition metals including $\mathrm{Fe}^{2+}$, 

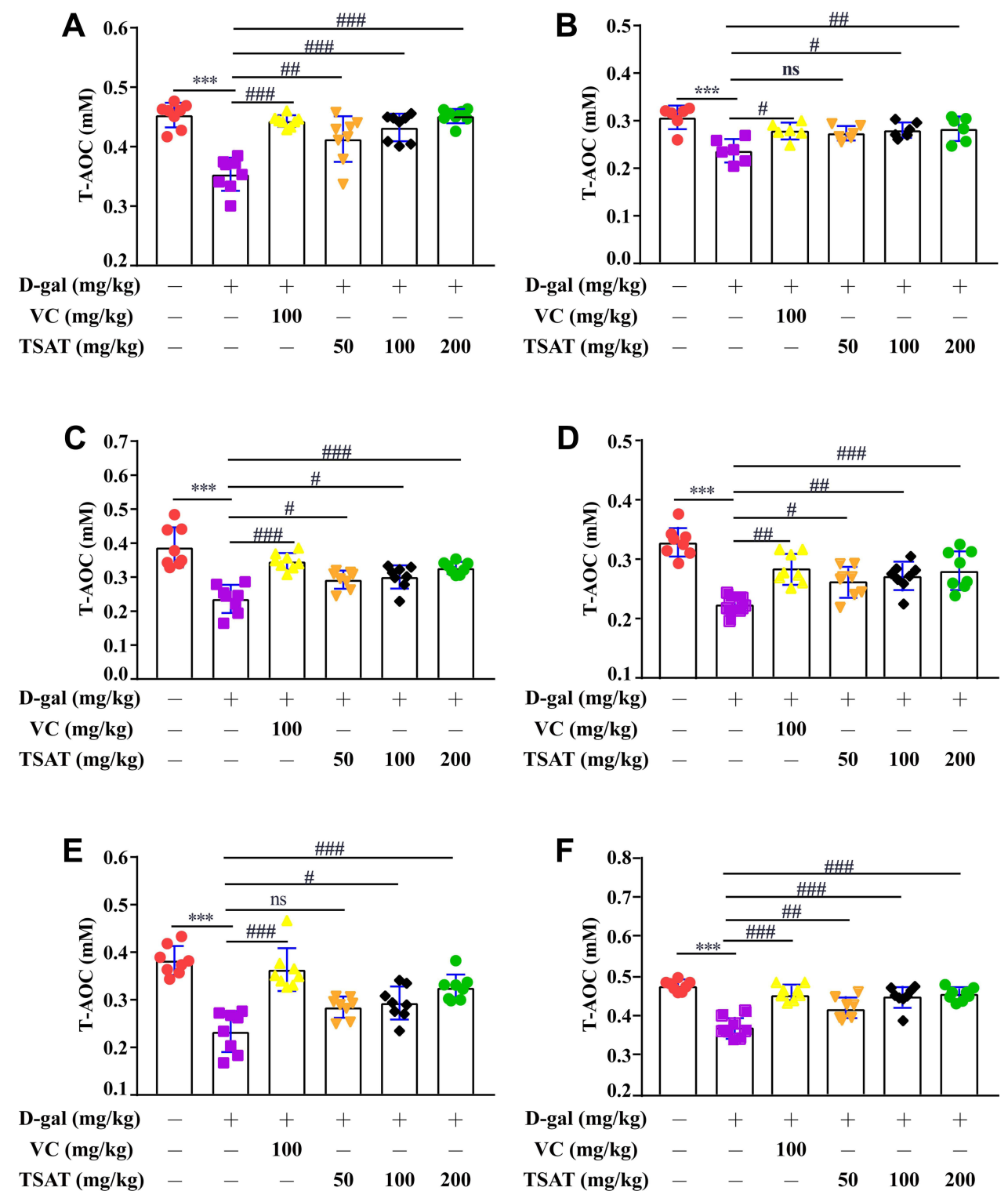

Figure 9 Effect of TSAT on T-AOC levels in (A) Serum, (B) Brain, (C) Heart, (D) Lung, (E) Spleen and (F) Kidney. ${ }^{*} * *_{\mathrm{p}}<0.000 \mathrm{I}$ vs normal group; ${ }^{\#} \mathrm{p}<0.05$, ${ }^{\#}{ }_{\mathrm{p}}<0.01$ and ${ }_{p}<0.0001$ vs model group. All the data are expressed as the means \pm SD of $6-8$ rats in each group.
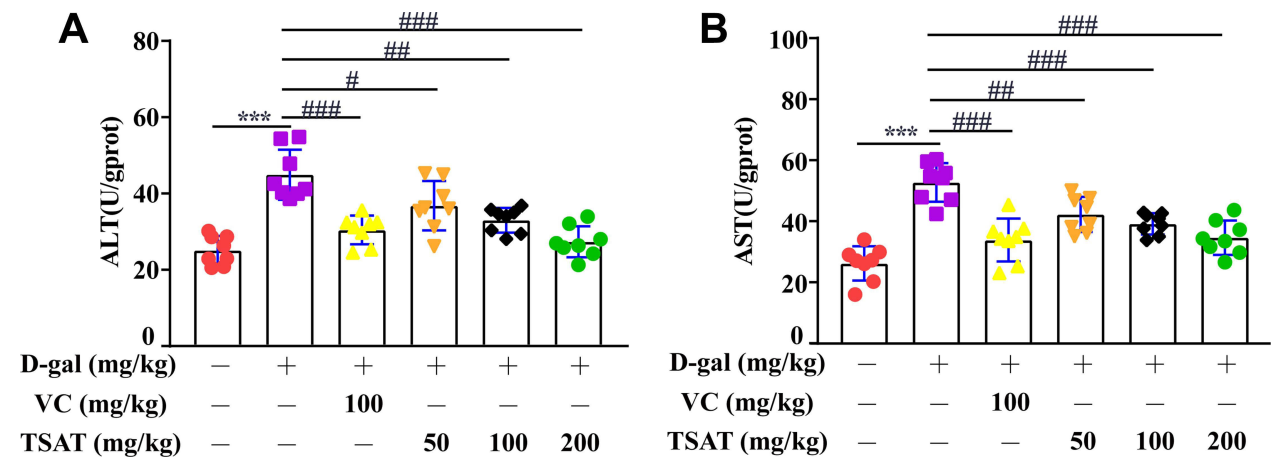

Figure 10 Effect of TSAT on (A) ALT and (B) AST levels in liver. $* * * *_{p}<0.0001$ vs normal group; ${ }^{\#}<0.05,{ }^{m}{ }_{p}<0.01$ and ${ }^{\# \#}{ }_{p}<0.0001$ vs model group. All the data are expressed as the means \pm SD of 6-8 rats in each group. 

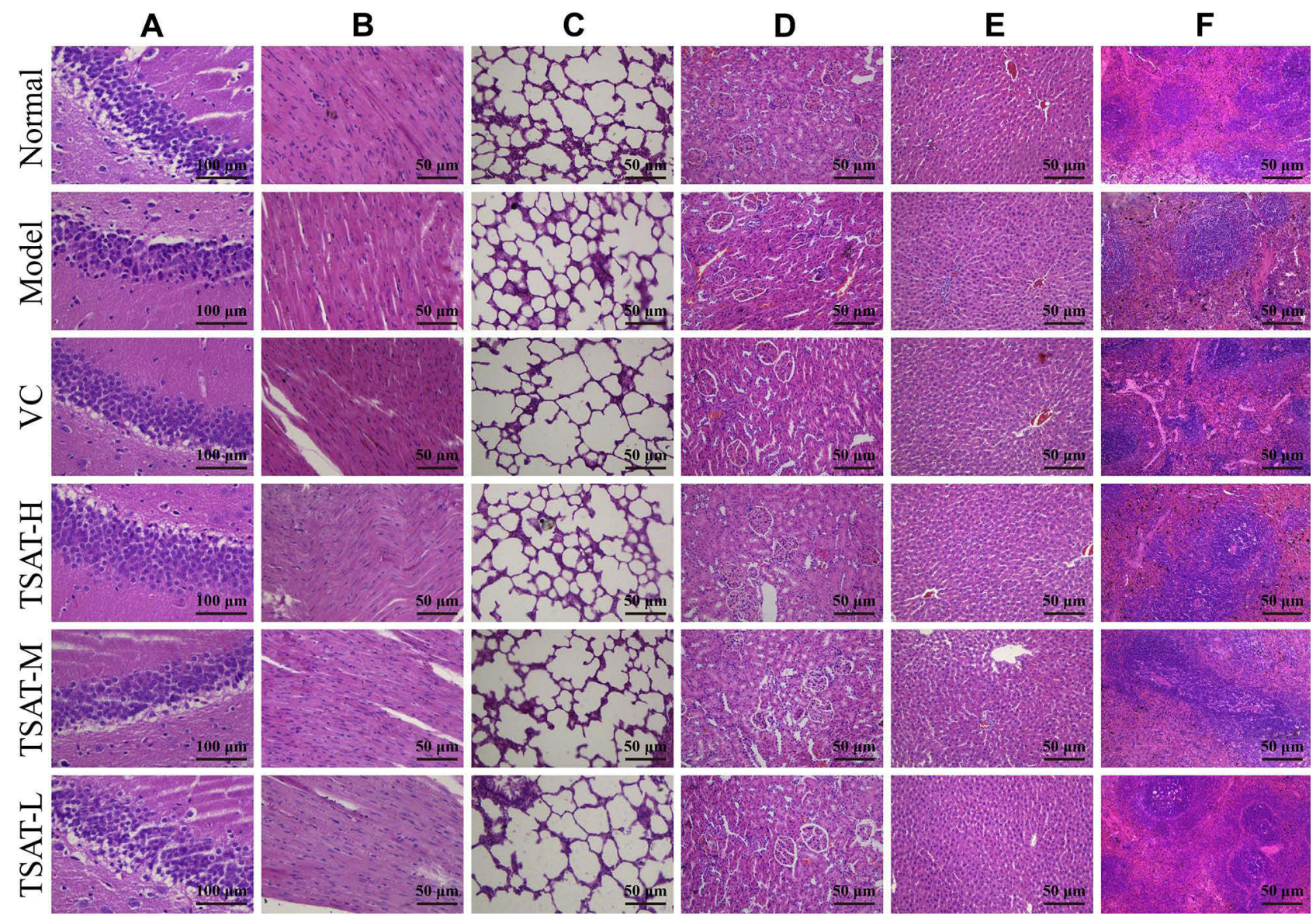

Figure I I Effects of TSAT on organ injury in the (A) Brain, (B) Heart, (C) Lung, (D) Kidney, (E) Liver and (F) Spleen in D-gal-induced aging rats ( $\mathrm{n}=3$ ).

Abbreviations: VC, rat treated with vitamin C (100 mg/kg); TSAT-H, rat treated with high concentration of TSAT (200 mg/kg); TSAT-M, rat treated with medium concentration of TSAT $(100 \mathrm{mg} / \mathrm{kg})$; TSAT-L, rat treated with low concentration of TSAT $(50 \mathrm{mg} / \mathrm{kg})$ (Haematoxylin/eosin staining, brain magnified at $400 X$, all other organs magnified at 200X).

$\mathrm{Cu}^{2+}$ and $\mathrm{Co}^{2+}$ can trigger the free radical reactions in magnifying the cellular damages. Among these transition metals, $\mathrm{Fe}^{2+}$ is known as the most powerful prooxidant on stimulating the lipid peroxidation by generating hydroxyl radicals through Fenton reaction. ${ }^{42} \cdot \mathrm{O}^{2-}$ is a product of biological and photochemical stimuli. It is highly toxic when combined with $\mathrm{HO} \cdot$, causing lipid peroxidation and damage to the normal structure of cells. ${ }^{43}$ Hence, the antioxidant and anti-aging properties of TSAT may be due to its free radical scavenging, tyrosinase inhibition and metal ion chelating. Additionally, there has report that the scavenging properties of TSAT were mainly associated with the structural differences such as the category and the sequence of the oligosaccharide chain at C-3 position in such saponins. ${ }^{20}$

Learning and memory deficits are considered to be clinical manifestations of neurodegenerative diseases associated with aging. ${ }^{26}$ Increasing evidence suggests that oxidative stress in the brain may play a key role in the pathological process of cognitive decline. ${ }^{44}$ This was confirmed in the MWM test study, where our results showed that escape latencies were longer in a D-gal-induced aging rat model, suggesting that D-gal impaired spatial learning in rats. Furthermore, the results across plateau counts also demonstrate that TSAT treatment improves spatial memory impairment in the D-gal-induced model. Moreover, cellular damage to hippocampal neurons has been shown to contribute to cognitive decline in $\mathrm{AD}$, and we used H\&E to evaluate the effects of TSAT on hippocampal neurons. ${ }^{45}$ Our results suggest that oral administration of TSAT has a neuroprotective effect, improving learning memory capacity and attenuating neuronal damage. This is consistent with a previous result that Aralia taibaiensis total saponin attenuates I/R-induced mitochondrial dysfunction and oxidative stress, thereby protecting brain cells from damage. ${ }^{46}$

Organ indices are indicators to assess the health status of animals. ${ }^{47}$ With aging, the body's immune system 
undergoes degenerative changes that not only reduce the sensitivity of non-specific immunity but also present a general imbalance in immune function, which ultimately leads to the development of diseases. Therefore, body immunity is one of the main tools in anti-aging research. The thymus and spleen are two important immune organs in the body, and their organ indices can initially reflect the strength of the nonspecific immune system. ${ }^{48,49}$ Compared with the normal group, the organ indices of the thymus and spleen in the model group of rats were reduced. However, there was an overall increase in organ indices in the drug group, but the variability was not significant, which may be related to the individual variability of the rats. The liver is also considered to be the immune organ of the animal, and alterations in the liver index can affect the body's immune function. ${ }^{50}$ The results showed that TSAT significantly increased the D-gal-induced decrease in the organ index, which is consistent with its traditional hepatoprotective effect. These results suggest that $\mathrm{D}$-gal successfully induces oxidative aging in rats, leading to a reduction in the organ index and that TSAT produces anti-aging effects by regulating immune organs.

It is well established that antioxidant enzyme activity decreases during aging. In the present study, SOD activity was significantly reduced during D-gal-induced aging. As the most important enzyme in living organisms, SOD protects the structural integrity of cells by blocking damage to cells by $\cdot \mathrm{O}^{2-}$. In a state of oxidative stress, the inward flow of intracellular $\mathrm{Ca}^{2+}$ leads to the irreversible conversion of xanthine dehydrogenase to xanthine oxidase, which in turn catalyses the oxidation of hypoxanthine, generating large amounts of $\cdot \mathrm{O}^{2-}$ that damage cellular structure and accelerate aging. ${ }^{51}$ Free radical overload leads to an overconsumption of SOD and reduced activity. CAT is another important antioxidant enzyme that maintains $\mathrm{H}_{2} \mathrm{O}_{2}$ homeostasis in the body, rapidly breaking down $\mathrm{H}_{2} \mathrm{O}_{2}$ into $\mathrm{H}_{2} \mathrm{O}$ and $\mathrm{O}_{2}$ and preventing abnormal accumulation of $\mathrm{H}_{2} \mathrm{O}_{2}$ in cells. The main role of GSH-Px is to interrupt the chain reaction of lipid peroxidation and catalyse the conversion of lipid peroxides into nontoxic hydroxyl compounds. ${ }^{28}$ In this study, we found that CAT and GSH-Px levels were significantly lower in the serum and organs of aged rats than in those of normal rats. This has been reported that this may be related to increased utilization of CAT and GSH-Px by the body's antioxidant system. ${ }^{10}$ MDA is a product of free radical chain reaction-induced lipid peroxidation and has been used as a marker to assess oxidative damage. In the current study, free radical action on cell membranes leading to membrane lipid damage may be responsible for the significant increase in MDA levels in the serum and organs of aging rats. ${ }^{10,51}$ The results of this study are consistent with earlier reports in the literature. We found that when TSAT was administered to aging rats, the levels of SOD, CAT and GSH-Px were significantly increased, and the levels of MDA were significantly decreased. This may be due to the active components of TSAT after metabolism in vivo, which restores the activity of these enzymes by providing single electrons to pair with free radicals, thereby scavenging them. This restoration contributes to cellular integrity, reduces oxidative stress and mitigates lipid peroxidation. These results strongly confirm the ability of TSAT to delay aging by scavenging free radicals, which was consistent with the results of in vitro experiments. Compared to single antioxidants, T-AOC can reflect the collective effect of enzymatic or nonenzymatic systems involved in antioxidant defence in biological fluids and is a comprehensive measure to evaluate the total antioxidant capacity of the body. ${ }^{52}$ Thus, higher levels of T-AOC may indicate an increased protective response of individuals and decreased susceptibility to oxidative damage.$^{53}$ In this study, the level of T-AOC decreased in rats exposed to D-gal for a long time, which may be due to the long-term consumption of antioxidant enzymes caused by high levels of oxidative stress, which was reversed by oral TSAT. The liver is the most vigorous metabolic organ in the body and an important detoxification organ. It plays an important role in regulating the health of the body. ${ }^{54}$ ALT is mainly found in the hepatocyte cytoplasm, while AST is mainly found in the mitochondria of hepatocytes. When liver cells are damaged, serum ALT levels increase, and when liver cells die, serum AST levels increase significantly. ${ }^{55}$ Therefore, serum ALT and AST levels can reflect the extent of liver injury. The results showed that ALT and AST levels were elevated in liver tissue after D-gal injury, but TSAT intervention inhibited the elevation of these transaminases. These results strongly suggest that TSAT attenuates oxidative damage to organs during aging to some extent by inhibiting oxidative stress. These can be demonstrated in histological observations.

\section{Conclusion}

In this study, we consolidated the antioxidant, anti-aging and organ protective effects of TSAT in vitro and in vivo. TSAT is a class of natural saponins with good scavenging effects on DPPH, ABTS, hydroxyl radicals and superoxide anion radicals and inhibits tyrosinase activity. In addition, TSAT exerted anti-aging effects in D-gal-induced aging rats by improving learning and memory capacity, 
preventing immune organ atrophy, increasing the activity of key antioxidant enzymes in the serum, brain, heart, lung, spleen and kidney, attenuating lipid peroxidation and inhibiting transaminase levels in liver tissues, thereby reducing oxidative and aging damage and exerting antiaging effects. However, although our study is important in consolidating the antioxidant, anti-aging and organ protective effects of TSAT, its molecular regulation on key antioxidant enzymes needs to be further investigated. Furthermore, due to the use of total saponins in this study, it should be further explored which specific components of TSAT contribute to these effects. Further investigation of these effects will provide supportive preclinical evidence for the potential clinical application of TSAT as a product for the treatment of age-related diseases.

\section{Acknowledgments}

This study was supported by the National Natural Science Foundation of China (No. 81703925) and the disciplinary innovation team construction project of Shaanxi University of Traditional Chinese Medicine (2019-YL11).

\section{Disclosure}

The authors report no conflicts of interest in this work.

\section{References}

1. Zhao H, Han Z, Li G, et al. Therapeutic potential and cellular mechanisms of panax notoginseng on prevention of aging and cell senescence-associated diseases. Aging Dis. 2017;8(6):721-739. doi:10.14336/ad.2017.0724

2. Liu Y, Weng W, Gao R, et al. New insights for cellular and molecular mechanisms of aging and aging-related diseases: herbal medicine as potential therapeutic approach. Oxid Med Cell Longev. 2019;20 19:4598167. doi:10.1155/2019/4598167

3. Harman D. Free radical theory of aging: an update: increasing the functional life span. Ann N Y Acad Sci. 2006;1067(1):10-21. doi:10. 1196/annals. 1354.003

4. Liochev S. Reactive oxygen species and the free radical theory of aging. Free Radic Biol Med. 2013;60:1-4. doi:10.1016/j. freeradbiomed.2013.02.011

5. Angelova P, Abramov A. Functional role of mitochondrial reactive oxygen species in physiology. Free Radic Biol Med. 2016;100:81-85. doi:10.1016/j.freeradbiomed.2016.06.005

6. Hewitt G, Jurk D, Marques F, et al. Telomeres are favoured targets of a persistent DNA damage response in ageing and stress-induced senescence. Nat Commun. 2012;3:708. doi:10.1038/ncomms 1708

7. Kregel K, Zhang H. An integrated view of oxidative stress in aging: basic mechanisms, functional effects, and pathological considerations. Am J Physiol Regul Integr Comp Physiol. 2007;292(1):R18-R36. doi:10.1152/ajpregu.00327.2006

8. Butterfield D, Abdul H, Opii W, et al. Pin1 in Alzheimer's disease. $J$ Neurovirol. 2006;98(6):1697-1706. doi:10.1111/j.1471-4159.2006. 03995.x

9. Seven A, Güzel S, Aslan M, et al. Lipid, protein, DNA oxidation and antioxidant status in rheumatoid arthritis. Clin Biochem. 2008;41(78):538-543. doi:10.1016/j.clinbiochem.2008.01.029
10. Li S, Liu M, Zhang C, et al. Purification, in vitro antioxidant and in vivo anti-aging activities of soluble polysaccharides by enzyme-assisted extraction from Agaricus bisporus. Int $\mathrm{J}$ Biol Macromol. 2018;109:457-466. doi:10.1016/j.ijbiomac.2017.12.108

11. Adefegha S, Okeke B, Oboh G. Antioxidant properties of eugenol, butylated hydroxylanisole, and butylated hydroxyl toluene with key biomolecules relevant to Alzheimer's diseases-in vitro. J Food Biochem. 2021;45(3):e13276. doi:10.1111/jfbc.13276

12. Yang X, Song W, Liu N, et al. Synthetic phenolic antioxidants cause perturbation in steroidogenesis in vitro and in vivo. Environ Sci Technol. 2018;52(2):850-858. doi:10.1021/acs.est.7b05057

13. Tang H, Yi Y, Wang Z, et al. [Studies on the triterpenoid saponins of the root bark of Aralia taibaiensis]. Yao Xue Xue Bao. 1996;31 (7):517-523. Chinese.

14. Xi M, Hai C, Tang H, et al. Antioxidant and antiglycation properties of triterpenoid saponins from Aralia taibaiensis traditionally used for treating diabetes mellitus. Redox Rep. 2010;15(1):20-28. doi:10.11 79/174329210x12650506623041

15. Cui J, Li Y, Jia N, et al. Insulin-secretagogue activity of eleven plant extracts and twelve pure compounds isolated from Aralia taibaiensis. Life Sci. 2013;92(2):131-136. doi:10.1016/j.1fs.2012.11.014

16. Weng Y, Yu L, Cui J, et al. Antihyperglycemic, hypolipidemic and antioxidant activities of total saponins extracted from Aralia taibaiensis in experimental type 2 diabetic rats. J Ethnopharmacol. 2014;152 (3):553-560. doi:10.1016/j.jep.2014.02.001

17. Wu Z, Li W, Zhou J, et al. Centella asiaticaOleanane- and ursane-type triterpene saponins from exhibit neuroprotective effects. J Agric Food Chem. 2020;68(26):6977-6986. doi:10.1021/acs.jafc. $0 \mathrm{c} 01476$

18. Tapondjou L, Nyaa L, Tane P, et al. Cytotoxic and antioxidant triterpene saponins from Butyrospermum parkii (Sapotaceae). Carbohydr Res. 2011;346(17):2699-2704. doi:10.1016/j.carres.2011. 09.014

19. Li Y, Guo Y, Xi M, et al. Saponins from Aralia taibaiensis attenuate D-galactose-induced aging in rats by activating FOXO3a and Nrf2 pathways. Oxid Med Cell Longev. 2014;2014:320513. doi:10.1155/ 2014/320513

20. Bi L, Tian X, Dou F, et al. New antioxidant and antiglycation active triterpenoid saponins from the root bark of Aralia taibaiensis. Fitoterapia. 2012;83(1):234-240. doi:10.1016/j.fitote.2011.11.002

21. Li C, He J, Yang Y, et al. White tip silver needle (slightly fermented white tea) flavonoids help prevent aging via antioxidative and anti-inflammatory effects. Drug Des Devel Ther. 2021;15:14 41-1457. doi:10.2147/dddt.S304885

22. Zhong S, Ge Q, Qu R, et al. Paeonol attenuates neurotoxicity and ameliorates cognitive impairment induced by d-galactose in ICR mice. J Neurol Sci. 2009;277(1-2):58-64. doi:10.1016/j.jns.2008.10.008

23. Chatatikun $\mathrm{M}$, Chiabchalard A. Thai plants with high antioxidant levels, free radical scavenging activity, anti-tyrosinase and anti-collagenase activity. BMC Complement Altern Med. 2017;17 (1):487. doi:10.1186/s12906-017-1994-7

24. Rafique R, Khan K, Chigurupati S, et al. Synthesis, in vitro $\alpha$ amylase inhibitory, and radicals (DPPH \& ABTS) scavenging potentials of new N-sulfonohydrazide substituted indazoles. Bioorg Chem. 2020;94:103410. doi:10.1016/j.bioorg.2019.103410

25. Morais D, Costa M, Santa Bárbara M, et al. Antioxidant, photoprotective and inhibitory activity of tyrosinase in extracts of Dalbergia ecastaphyllum. PLoS One. 2018;13(11):e0207510. doi:10.1371/journal.pone.0207510

26. Liang J, Wu Y, Yuan H, et al. Dendrobium officinale polysaccharides attenuate learning and memory disabilities via anti-oxidant and anti-inflammatory actions. Int J Biol Macromol. 2019;126:414-426. doi:10.1016/j.ijbiomac.2018.12.230

27. Min F, Sun H, Wang B, et al. Hepatoprotective effects of hydroxysafflor yellow A in D-galactose-treated aging mice. Eur J Pharm Sci. 2020;881:173214. doi:10.1016/j.ejphar.2020.173214 
28. Liu B, Ma R, Zhang J, et al. Preventive effect of small-leaved kuding tea (Ligustrum robustum (Roxb.) B1.) polyphenols on D-galactoseinduced oxidative stress and aging in mice. Evid Based Complement Alternat Med. 2019;2019:3152324. doi:10.1155/2019/3152324

29. Xu X-Y, Zheng J, Meng J-M, et al. Effects of food processing on in vivo antioxidant and hepatoprotective properties of green tea extracts. Antioxidants. 2019;8(12):572. doi:10.3390/antiox8120572

30. Li G, Yu J, Zhang L, et al. Onjisaponin B prevents cognitive impairment in a rat model of D-galactose-induced aging. Biomed Pharmacother. 2018;99:113-120. doi:10.1016/j.biopha.2018.01.006

31. Li L, Xu M, Shen B, et al. Moderate exercise prevents neurodegeneration in D-galactose-induced aging mice. Neural Regen Res. 2016;11(5):807-815. doi:10.4103/1673-5374.182709

32. Wang M, Tian Y, Du -Y-Y, et al. Protective effects of Araloside $\mathrm{C}$ against myocardial ischaemia/reperfusion injury: potential involvement of heat shock protein 90. J Cell Mol Med. 2017;21(9): 1870-1880. doi:10.1111/jcmm.13107

33. Wang S, Zeng M, Li B, et al. Raw and salt-processed Achyranthes bidentata attenuate LPS-induced acute kidney injury by inhibiting ROS and apoptosis via an estrogen-like pathway. Biomed Pharmacother. 2020;129:110403. doi:10.1016/j.biopha.2020.110403

34. Wang Y, Li Y, He C, et al. Mitochondrial regulation of cardiac aging. Biochim Biophys Acta Mol Basis Dis. 2019;1865(7):1853-1864. doi:10.1016/j.bbadis.2018.12.008

35. Du Y, Wang M, Liu X, et al. Araloside C prevents hypoxia/reoxygenation-induced endoplasmic reticulum stress via increasing heat shock protein 90 in $\mathrm{H} 9 \mathrm{c} 2$ cardiomyocytes. Front Pharmacol. 2018;9:180. doi:10.3389/fphar.2018.00180

36. Chan M, Xu D, Guo L, et al. Cyanine fluorophores for cellular protection against ROS in stimulated macrophages and two-photon ROS detection. Org Biomol Chem. 2015;13(26):7307-7312. doi:10. 1039/c5ob00643k

37. Martins D, Titorenko V, English A. Cells with impaired mitochondrial $\mathrm{H} 2 \mathrm{O} 2$ sensing generate less $\bullet \mathrm{OH}$ radicals and live longer. Antioxid Redox Signal. 2014;21(10):1490-1503. doi:10.1089/ars.20 13.5575

38. Amorati R, Menichetti S, Viglianisi C, et al. Proton-electron transfer pathways in the reactions of peroxyl and dpph radicals with hydrogen-bonded phenols. Chem Comm. 2012;48(97):11904-11906. doi: $10.1039 / \mathrm{c} 2 \mathrm{cc} 36531 \mathrm{f}$

39. Zolghadri S, Bahrami A, Hassan Khan M, et al. A comprehensive review on tyrosinase inhibitors. J Enzyme Inhib Med Chem. 2019;34 (1):279-309. doi:10.1080/14756366.2018.1545767

40. Hasegawa T. Tyrosinase-expressing neuronal cell line as in vitro model of parkinson's disease. Int J Mol Sci. 2010;11(3):1082-1089. doi:10.3390/ijms 11031082

41. Zhang H, Zhou Q, Hudson AO. Tyrosinase inhibitory effects and antioxidative activities of saponins from Xanthoceras Sorbifolia nutshell. PLoS One. 2013;8(8):e70090. doi:10.1371/journal.pone.00 70090

42. Sun YX, Kennedy JF. Antioxidant activities of different polysaccharide conjugates (CRPs) isolated from the fruiting bodies of Chroogomphis rutilus (Schaeff.: Fr.) O. K. Miller. Carbohydr Polym. 2010;82(2):510-514. doi:10.1016/j.carbpol.2010.05.010
43. Atif F, Kaur M, Ansari R, et al. Channa punctata brain metallothionein is a potent scavenger of superoxide radicals and prevents hydroxyl radical-induced in vitro DNA damage. $J$ Biochem Mol Toxicol. 2008;22(3):202-208. doi:10.1002/jbt.20230

44. Ruan Q, Hu X, Ao H, et al. The neurovascular protective effects of huperzine A on D-galactose-induced inflammatory damage in the rat hippocampus. Gerontology. 2014;60(5):424-439. doi:10.1159/000 358235

45. Huang C, Garnet IM, Chun W, et al. Evidence of the impact of systemic inflammation on neuroinflammation from a non-bacterial endotoxin animal model. J Neuroinflammation. 2018;15(1):147. doi:10.1186/s12974-018-1163-Z

46. Duan J, Cui J, Zheng H, et al. Aralia taibaiensis protects against I/ R-induced brain cell injury through the Akt/SIRT1/FOXO3a pathway. Oxid Med Cell Longev. 2019;2019:1-18. doi:10.1155/ 2019/7609765

47. Tao T, He B. Treatment of d-galactose induced mouse aging with Lycium barbarum polysaccharides and its mechanism study. Afr J Tradit Complement Altern Med. 2013;10(4):12-17. doi:10.4314/ ajtcam.v10i4.3

48. Xiong W. Research on effect of ginkgo aglucone flavone to human body organs and immune function. Pak J Pharm Sci. 2014;27(4 Suppl):1099-1102.

49. Chen NY, Liu CW, Wei L, et al. Extract of fructus cannabis ameliorates learning and memory impairment induced by D-galactose in an aging rats model. Evid Based Complement Alternat Med. 2017; 2017:1-13. doi:10.1155/2017/4757520

50. Khan SS, Singer BD, Vaughan DE. Molecular and physiological manifestations and measurement of aging in humans. Aging Cell. 2017;16(4):624-633. doi:10.1111/acel.12601

51. Ramesh T, Kim S, Sung J, et al. Effect of fermented panax ginseng extract (GINST) on oxidative stress and antioxidant activities in major organs of aged rats. Exp Gerontol. 2012;47(1):77-84. doi:10. 1016/j.exger.2011.10.007

52. Farhat Z, Browne R, Bonner M, et al. How do glutathione antioxidant enzymes and total antioxidant status respond to air pollution exposure? Environ Int. 2018;112:287-293. doi:10.1016/j.envint.2017. 12.033

53. Wang M, Utell MJ, Schneider A, et al. Does total antioxidant capacity modify adverse cardiac responses associated with ambient ultrafine, accumulation mode, and fine particles in patients undergoing cardiac rehabilitation? Environ Res. 2016;149:15-22. doi:10.1016/j. envres.2016.04.031

54. Zhao X, Wang H, Yang Y, et al. Protective effects of silymarin against D-Gal/LPS-induced organ damage and inflammation in mice. Drug Des Devel Ther. 2021;15:1903-1914. doi:10.2147/dddt. S305033

55. Ozer JS, Chetty R, Kenna G, et al. Enhancing the utility of alanine aminotransferase as a reference standard biomarker for drug-induced liver injury. Regul Toxicol Pharmacol. 2010;56(3):237-246. doi:10. 1016/j.yrtph.2009.11.001

\section{Publish your work in this journal}

Drug Design, Development and Therapy is an international, peerreviewed open-access journal that spans the spectrum of drug design and development through to clinical applications. Clinical outcomes, patient safety, and programs for the development and effective, safe, and sustained use of medicines are a feature of the journal, which has also been accepted for indexing on PubMed Central. The manuscript management system is completely online and includes a very quick and fair peer-review system, which is all easy to use. Visit http://www. dovepress.com/testimonials.php to read real quotes from published authors. 